\title{
In vivo imaging with a flat, lensless microscope
}

\section{Jesse Adams}

Rice University https://orcid.org/0000-0002-6767-3221

\section{Dong Yan}

Rice University https://orcid.org/0000-0002-5235-8501

Jimin Wu

Rice University

Vivek Boominathan

Rice University

Sibo Gao

Rice University

\section{Alexander Rodriguez}

Rice University

\section{Jennifer Carns}

Rice University

\section{Rebecca Richards-Kortum}

Rice University

\section{Caleb Kemere}

Rice University https://orcid.org/0000-0003-2054-0234

\section{Ashok Veeraraghavan}

Rice University https://orcid.org/0000-0001-5043-7460

Jacob Robinson ( $\nabla$ jtrobinson@rice.edu )

Rice University

\section{Article}

Keywords: microscopy, imaging, lensless microscope

Posted Date: January 20th, 2021

DOI: https://doi.org/10.21203/rs.3.rs-142332/v1

License: (c) (1) This work is licensed under a Creative Commons Attribution 4.0 International License. Read Full License 
Version of Record: A version of this preprint was published at Nature Biomedical Engineering on March 7th, 2022. See the published version at https://doi.org/10.1038/s41551-022-00851-z. 
1 In vivo imaging with a flat, lensless microscope

\author{
2 Jesse K. Adams, ${ }^{\dagger, 1,2}$ Dong Yan, ${ }^{\dagger, 1,2}$ Jimin Wu, ${ }^{\dagger, 4}$ ViVeK BOOMINATHAn, ${ }^{2}$ \\ 3 Sibo Gao, ${ }^{2,3}$ Alex V. Rodriguez, ${ }^{2}$ Jennifer Carns, ${ }^{4}$ Rebecca \\ 4 Richards-Kortum, ${ }^{2,4}$ Caleb Kemere, ${ }^{2,3,4}$ AshoK Veeraraghavan, ${ }^{1,2,5,{ }^{*}}$ \\ 5 JACOB T. ROBINSON ${ }^{1,2,3,4, *}$

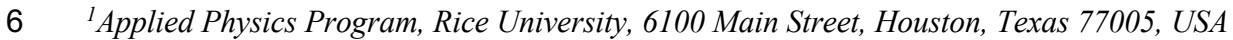 \\ $7 \quad{ }^{2}$ Department of Electrical and Computer Engineering, Rice University, 6100 Main Street, Houston, \\ 8 Texas 77005, USA \\ $9{ }^{3}$ Department of Neuroscience, Baylor College of Medicine, One Baylor Plaza, Houston, Texas 77030, \\ 10 USA \\ $11{ }^{4}$ Department of Bioengineering, Rice University, 6100 Main Street, Houston, Texas 77005, USA \\ 125 Department of Computer Science, Rice University, 6100 Main Street, Houston, Texas 77005, USA \\ $13 \dagger$ these authors contributed equally \\ 14 *vashok@rice.edu; ;trobinson@rice.edu
}

Abstract:

Lenless microscopes that form images using simple, compact optics, along with associated computational algorithms provide several advantages: small, inexpensive form factors, large fields of view (FOV), and the ability to refocus an image after capture. To date, however, lensless microscopy has yet to be demonstrated for in vivo biological imaging. The primary challenge with in vivo bio-imaging is these dense, low-contrast scenes cannot be accurately reconstructed with existing lensless techniques. To overcome this challenge, we designed an optical phase mask that creates a point-spread function consisting of high-contrast contours with a broad spatial frequency spectrum. Here we show that this "contour" phase mask enables in vivo imaging where previous lensless imaging technologies have failed. As a proof of concept, we demonstrate 3D imaging of a behaving invertebrate, functional calcium imaging in mouse cortex, and in vivo imaging of microvasculature in the human oral mucosa. Together these results demonstrate the ability of lensless imaging to operate in realistic biological imaging applications ranging from pre-clinical animal models to clinical biomedical imaging. Overall, this "Bio-FlatScope" is a clinically relevant imaging technique with advantages of 3D computational refocusing in a low-cost, small form factor that is useful for low-resource settings and difficult-to-reach areas of the body.

\title{
Main
}

Computational imaging algorithms, co-designed with optics and sensors, open up new design degrees of freedom for imaging systems [1-3]. For example, computational imaging techniques have already made a significant impact on microscopy, for applications like superresolution [4,5], phase imaging [6], and three-dimensional imaging [7,8]. Taken to an extreme, computation can completely replace the traditional lens(es) of an optical system [9], or be combined with light-modulating masks $[10,11]$. Unlike lens-based imaging systems where the goal is to project a reproduction of a (potentially magnified) scene onto an image sensor, a lensless imaging system seeks to produce an invertible transfer function between the incident light field and the sensor measurements. These measurements often may not resemble a traditional image [12-18] but contain sufficient information for a computational algorithm to reconstruct an image. 
By removing lenses from the image capture process, systems can achieve substantial improvements in the FOV, preform three-dimensional capture and refocusing, capture light with high-efficiency, and be miniaturized into small form factors [10-12,19].

Despite the transformative potential of lensless microscopy, there are currently no demonstrations of in vivo biological imaging due to the fact that biological scenes are dense, dim, and low contrast, which can often result in noisy reconstructed images. Indeed, previous lensless microscopy demonstrations have relied on sparse, bright, or high-contrast samples where strong regularization and/or deblurring can be used to reconstruct estimates of the original image $[10,19,20]$.

To address the challenge of biological imaging we designed a phase mask especially suited for the low-contrast challenges expected in biological imaging. The key feature of this phase mask is that it achieves a high-contrast and spatially localized sparse point spread function (PSF) that captures textural frequencies common in natural and biological samples (Fig 1d). To create this PSF we generate Perlin noise and apply uncanny edge detection [3]. Figure 1b shows the simulated modulation transfer function (MTF) of our contour-based PSF compared to other PSFs used in lensless imaging systems. The relatively flat MTF spectrum indicates that information from most spatial frequencies are well-preserved by the optical transfer function leading to improved image reconstruction for dense, low contrast samples like biological tissue. We chose to create this PSF using a phase mask (Fig. S2), because phase masks are capable of producing a wide variety of PSFs [21-26] and allow higher light throughput compared to amplitude masks (which block some of the incoming light) [10]. We refer to this prototype device (Fig 1a) as a "Bio-FlatScope" because it achieves a similar flat form factor as previously reported "FlatScopes" [10], but the new contour phase mask allows us to perform the first accurate lensless imaging of biological tissue in vivo. 

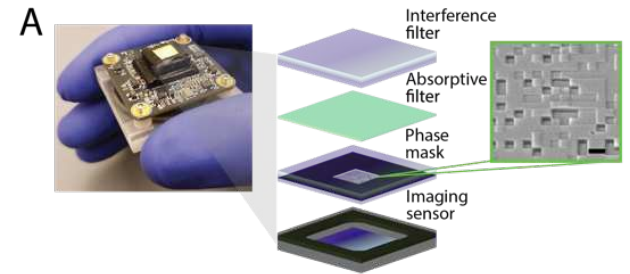

C

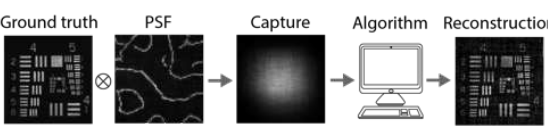

D
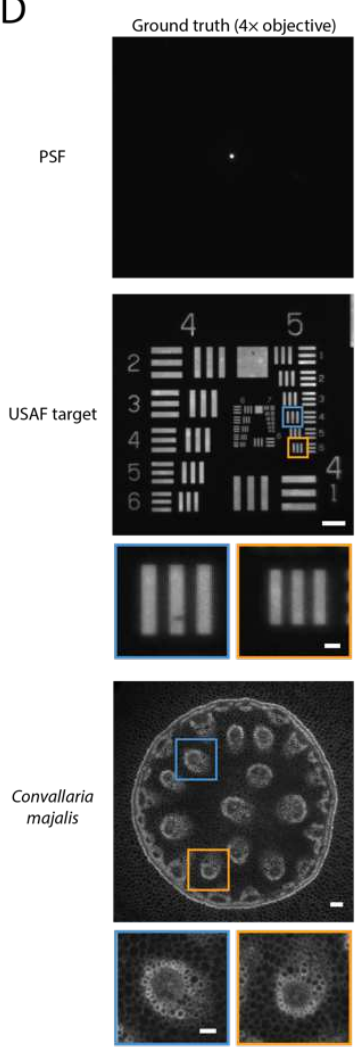

Bio-FlatScope
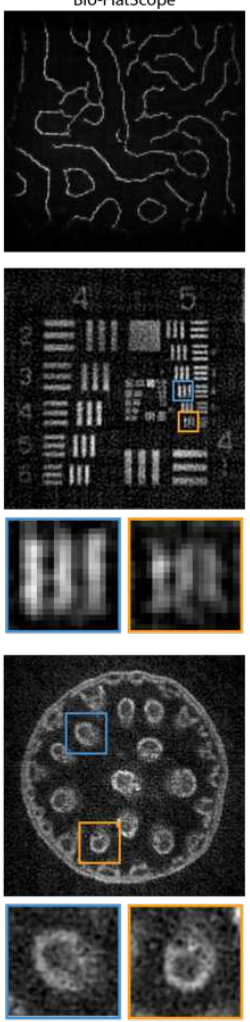
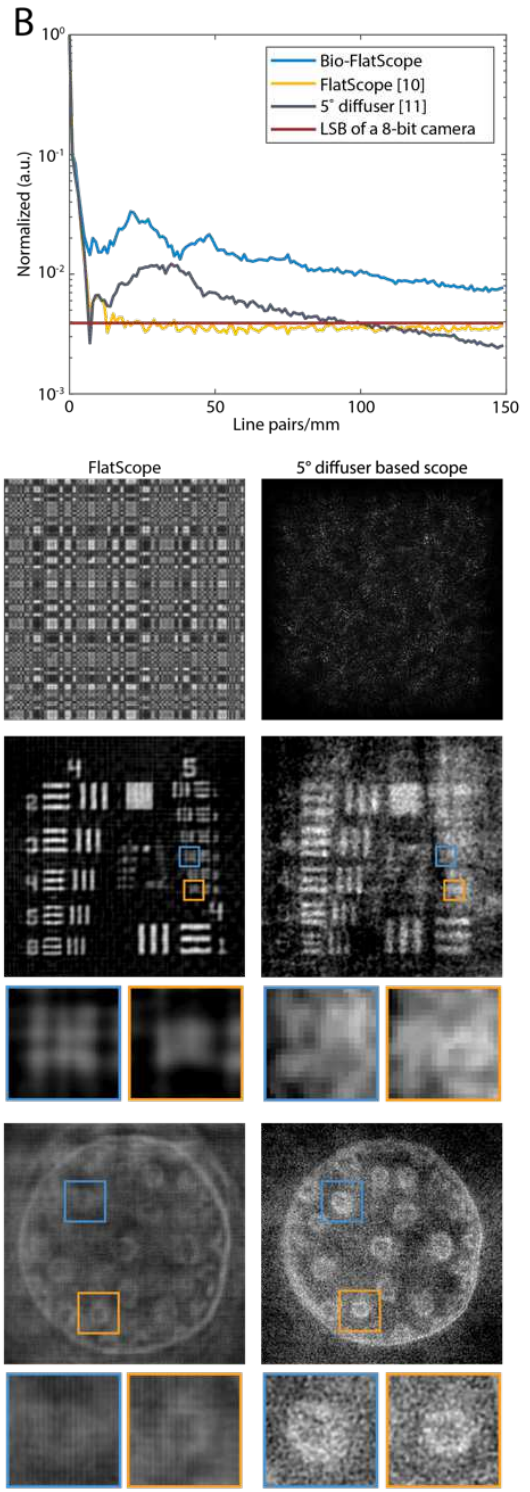

Fig. 1. Bio-FlatScope for in vivo fluorescence imaging. (A) Photo of a Bio-FlatScope prototype including the offthe-shelf board level camera. Zoom-in shows components that filter excitation light and apply the phase mask transfer function to the incident light. Also shown is a scanned electron micrograph of a portion of the fabricated phase mask. Scale bar $4 \mu \mathrm{m}$. (B) Modulation transfer function (MTF) comparison among PSF designs used by lensless imaging systems show that the contour PSF used for the Bio-FlatScope contains more high-frequency components, which contributes to improved performance. LSB: least significant bit. (C) Flow chart showing the imaging procedure of Bio-FlatScope. (D) High spatial resolution images of a USAF test target and fixed biological samples. Row 1: PSF captures of different lensless imaging systems. Row 2: Ground truth captured by an epifluorescence microscope and reconstructed by 3 different lensless imaging systems. While FlatScope and BioFlatScope both show good performance for the high-contrast USAF target, only the Bio-FlatScope performs well on the low-contrast, dense Convallaria sample. USAF 1951 Resolution test target scale bar, $100 \mu \mathrm{m}$. Insets below show zoom-ins of group 5, elements of $4 \& 6$. Scale bar, $10 \mu \mathrm{m}$. Convallaria majalis scale bar, $100 \mu \mathrm{m}$. Insets below show zoom-in comparisons. Scale bar, $50 \mu \mathrm{m}$. 


\section{Results}

To evaluate the performance of Bio-FlatScope, we constructed prototypes using Sony IMX178 monochromatic $6 \mathrm{MP}$ imaging sensors with $2.4 \mu \mathrm{m}$ pixels. Phase masks were fabricated using a two-photon lithography system (Nanoscribe, Photonic Professional GT2) for ease of prototyping (see Methods). The contour-based phase masks were designed with a minimum feature width of 6-12 $\mu \mathrm{m}$.

\section{Spatial Resolution \& Fixed Biological Samples}

Using USAF resolution targets, we found that reconstructed images taken by the Bio-FlatScope achieve $<9 \mu \mathrm{m}$ lateral resolution. We measured this resolution by capturing images of a negative 1951 USAF Resolution Target (Edmund Optics \#59-204) with an added fluorescent background. Multiple images were captured at $15 \mathrm{~ms}$ each and averaged to remove noise. Excitation light was provided in a near-epi configuration with a fiber/microprism (Fig S3). Images were captured at a distance of $\sim 4 \mathrm{~mm}$. In Fig. 1d, we show that we can achieve $<9 \mu \mathrm{m}$ resolution. While this spatial resolution is reduced by $\sim 1.5 \times-3.5 \times$ compared to (single-photon) miniature microscopes (see Table S1), we find that our system achieves cellular resolution under sparse labeling conditions.

The main advantage of the Bio-FlatScope is its ability to accurately reconstruct dense, dim, and low-contrast biological samples; thus, we compare the performance of Bio-FlatScope to other lensless imaging techniques for imaging a fluorescent biological sample. Specifically, we compared reconstructed images of Convallaria majalis (lily of the valley) stained with green fluorescent protein (GFP). We notice that for the high-contrast USAF test targets, both the FlatScope and Bio-FlatScope show clear image reconstructions; however, for the Convallaria, only the Bio-FlatScope shows good correspondence to ground truth images captured with a $4 \times$ microscope objective (Nikon Fluor) (Fig. 1d). These data are based on multiple images of the Convallaria slice (at $\sim 3.5 \mathrm{~mm}$ from the device) captured at $200 \mathrm{~ms}$ each in the near-epi configuration and averaged for noise removal. Note in the Convallaria insets that for the lensless approaches tested, only the Bio-FlatScope can resolve some of the large circular plant cells with sizes of around $10 \mu \mathrm{m}$. We also confirmed that Bio-FlatScope can resolve images in fixed neural tissue using ex vivo mouse brain slices (deep cortex and hippocampus) expressing the fluorescent protein GCaMP6f (see Methods). Here we capture 20 images at a distance of $\sim 5.3 \mathrm{~mm}$ using transmissive illumination with 1s exposures averaged to remove noise. Comparisons between Bio-FlatScope images and ground truth microscopy show clear delineation between cortex layer 2/3, corpus callosum and hippocampus (CA1) (Fig. S4). We can also identify clusters of neurons in the cortex as well as the pyramidal layer of CA1 (see far-right column of Fig. S4).

\section{Single capture three-dimensional imaging in vivo}

One significant advantage for lensless imaging is the ability to obtain three-dimensional information with a single image capture due to the fact that the PSFs change as a function of depth. To demonstrate that Bio-FlatScope is also capable of single-shot 3D reconstruction of biological samples we imaged Hydra vulgaris, a freshwater cnidarian polyp, which has a simple, yet dynamic network of neurons, optical transparency, and the ability to regenerate from a small patch of tissue [27,28]. An additional advantage of Hydra is their genetic tractability and a number of transgenic lines including lines that express GFP or calcium dependent fluorescent protein GCaMP7b [29], as well as their compatibility with microfluidic devices [27]. Using Bio-FlatScope we captured videos at $1 \mathrm{~Hz}$ of Hydra expressing GFP in interstitial cell lineage. We were then able to reconstruct these images in three dimensions (Fig. 2a) during post-processing using 


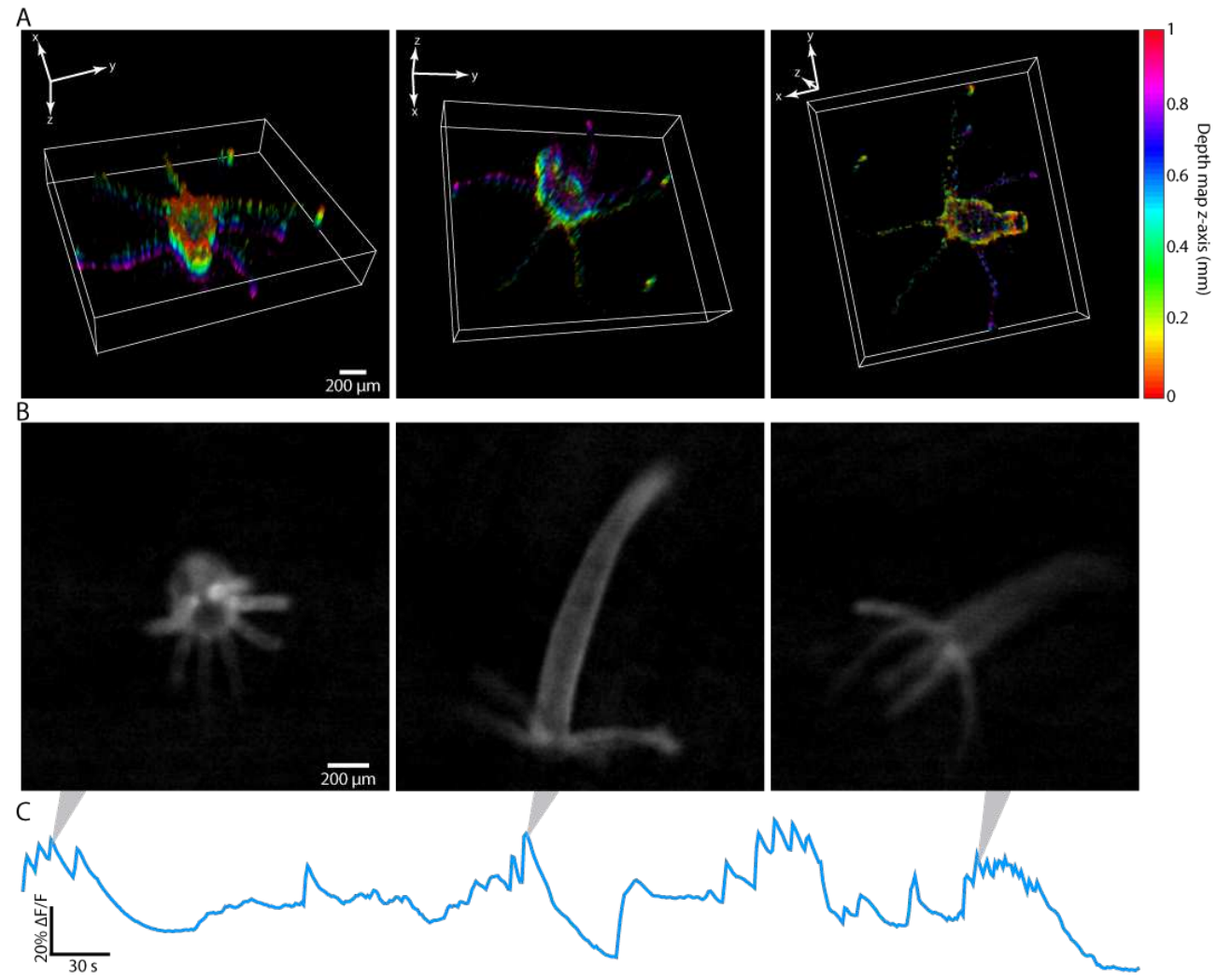

Fig. 2. $\mathrm{Ca}^{2+}$ imaging (GCaMP7b) and three-dimensional imaging (GFP) of Hydra vulgaris in vivo. (A) Select angles of three-dimensional Bio-FlatScope reconstructions of Hydra vulgaris expressing GFP in the interstitial cell lineage. Colors represent relative depth location along the z-axis. Scale bar, $200 \mu \mathrm{m}$. (B) Selected Bio-FlatScope reconstructed frames of video of Hydra vulgaris expressing GCaMP7b in muscle cells. Scale bar, $200 \mu \mathrm{m}$. (C) $\Delta \mathrm{F} / \mathrm{F}$ traces showing the $\mathrm{Ca}^{2+}$ responses over a 5-minute recording.

a series of PSFs corresponding to different distances from the sensor in $50 \mu \mathrm{m}$ increments. Here we show a $4 \mathrm{~mm}^{3}$ volume reconstructed from a single capture. By contrast traditional microscopes typically require scanning spatially, axially, or both. This single-capture, threedimensional imaging opens up the possibility for high-resolution, high-speed, volumetric imaging of whole organisms freely behaving over large FOVs.

\section{In vivo epifluorescence calcium imaging of whole animal with Hydra vulgaris}

Given the high-speed volumetric imaging made possible by single-shot 3D imaging, we tested the ability of Bio-FlatScope to capture whole-animal dynamic calcium activity in Hydra. We began by imaging Hydra vulgaris expressing GCaMP7b in muscle cells [30]. We captured video of Hydra freely behaving at $2 \mathrm{~Hz}$ over a FOV $>12 \mathrm{~mm}^{2}$. In Fig. 2b\&c we show selected frames from a video of the reconstruction as well as the overall change in fluorescence over time $(\Delta \mathrm{F} / \mathrm{F})$. The recordings show strong calcium responses during contraction events as reported previously [31] (full video shown in Visualization 1). Although movement of the animal can produce some noise in the $\Delta \mathrm{F} / \mathrm{F}$ signal, the deformable Hydra body makes it difficult to generate adaptive ROIs that move with the animal. Nevertheless, the bright synchronous calcium activity in the Hydra peduncle allows for fixed ROIs to effectively capture calcium dynamics [27,32]. Here the large FOV and 3D reconstructions help us capture movies of wholeanimal calcium dynamics, which can be used by neuroscientists to study information processing in these small model organisms [33]. 


\section{In vivo epifluorescence calcium imaging in mice}

We also found that the Bio-FlatScope is capable of imaging calcium dynamics in mouse cortex, which is commonly used for neuroscience experiments and preclinical testing [34-37]. This type of in vivo imaging is particularly difficult for lensless imaging and epifluorescence imaging because tissue scattering causes blurring and loss of contrast.

We hypothesized that the optimized contour PSF with high contrast features will provide good protection against noise amplification in these low-contrast scenes and allow BioFlatScope to perform reconstructions through scattering media similar to epifluorescence imaging (Fig. S5).

To test our hypothesis, mice expressing GCaMP6f in the motor cortex (see Methods) were head-fixed and placed upon a freely moving treadmill to allow for locomotion during video capture (Fig. 3a). We recorded calcium imaging movies over a FOV of $\sim 16 \mathrm{~mm}^{2}$ of the entire cranial window (Fig. S6) using a $473 \mathrm{~nm}$ excitation light source (see Methods). A cropped region near the injection site is shown in Figure $3 \mathrm{c}$. Compared to previously reported miniature microscopes with cellular resolution [38], we achieve more than a 30× increase in the FOV (see Table S1). We also measured the velocity of the treadmill during imaging sessions and synchronized the image captures with this velocity data. During the recording sessions, we applied a brushing tactile stimulation in $30 \mathrm{~s}$ intervals to encourage locomotion and neural response in the motor cortex. Correlation between locomotion (as performed by the mouse here on a treadmill) and neuronal response in the motor cortex has been established in prior reports [39-41] and is confirmed in our epifluorescence imaging. In the regions of high activity in the motor cortex, we observed peak fluorescence signals during locomotion an average of $\sim 3 \times$ greater than periods of no motion ( $\mathrm{rms}$ ) and were able to resolve blood vessels as small as $\sim 10 \mu \mathrm{m}$ in diameter. To compare our Bio-FlatScope imaging to conventional epifluorescence, we replaced the Bio-FlatScope with a $4 \times$ objective lens and fluorescence microscope. We performed epifluorescence imaging under the same conditions within 30 minutes of our BioFlatScope recordings (see Methods) and found very similar spatiotemporal dynamics in the two data sets (Fig. 3, Fig. S7 and S8). We chose the $4 \times$ objective lens to accommodate the FOV captured by Bio-FlatScope (while maintaining high resolution) and registered both data sets to accommodate for movement $[42,43]$.

To compare Bio-FlatScope data with epifluorescence data, we selected 4-second windows time-aligned to the response to stimulation (reflected as a large change in ambulatory activity of the mouse) over a two-minute period (Fig. 3d\&e, shown in green). Ambulatory activity was determined by synchronizing the wheel velocity with the imaging data for both Bio-FlatScope and epifluorescence (shown in Fig. 3d\&e for a single ROI, Visualization 2 shows video for multiple regions). We validated the similarity of the windowed $\Delta \mathrm{F} / \mathrm{F}$ responses with correlation, finding a median correlation coefficient of 0.852 with interquartile range (IQR) of 0.131 (Fig $3 \mathrm{f}$, left-side). We also compared data from time windows of little to no locomotion, where velocity $<1 \mathrm{~cm} / \mathrm{s}$ (windows shown in Fig. S7) with epifluorescence captured motion resulting in a median correlation coefficient of -0.375 and an IQR of 0.513 (Fig 3f, right-side). As expected, data from regions of motion/stimuli highly correlate when comparing Bio-FlatScope to epifluorescence data (coefficients range from 0.65 to $0.97, p<0.001$ ), while regions of little to no motion show more random correlation (coefficients ranging from -0.52 to 0.31 , two values of $p<0.001$ and six values of $p>0.05$ ), confirming that using Bio-FlatScope we can capture behaviorally relevant calcium dynamics that are comparable to traditional dynamics capture with conventional epifluorescence microscopes.

In addition, when plotting the time of the calcium peaks over the FOV we find that when the animal initiates movement the majority of early peaks appear in the bottom and right of the FOV and the majority of late peaks appear in the top and left (Fig. 3g). This pattern is also 

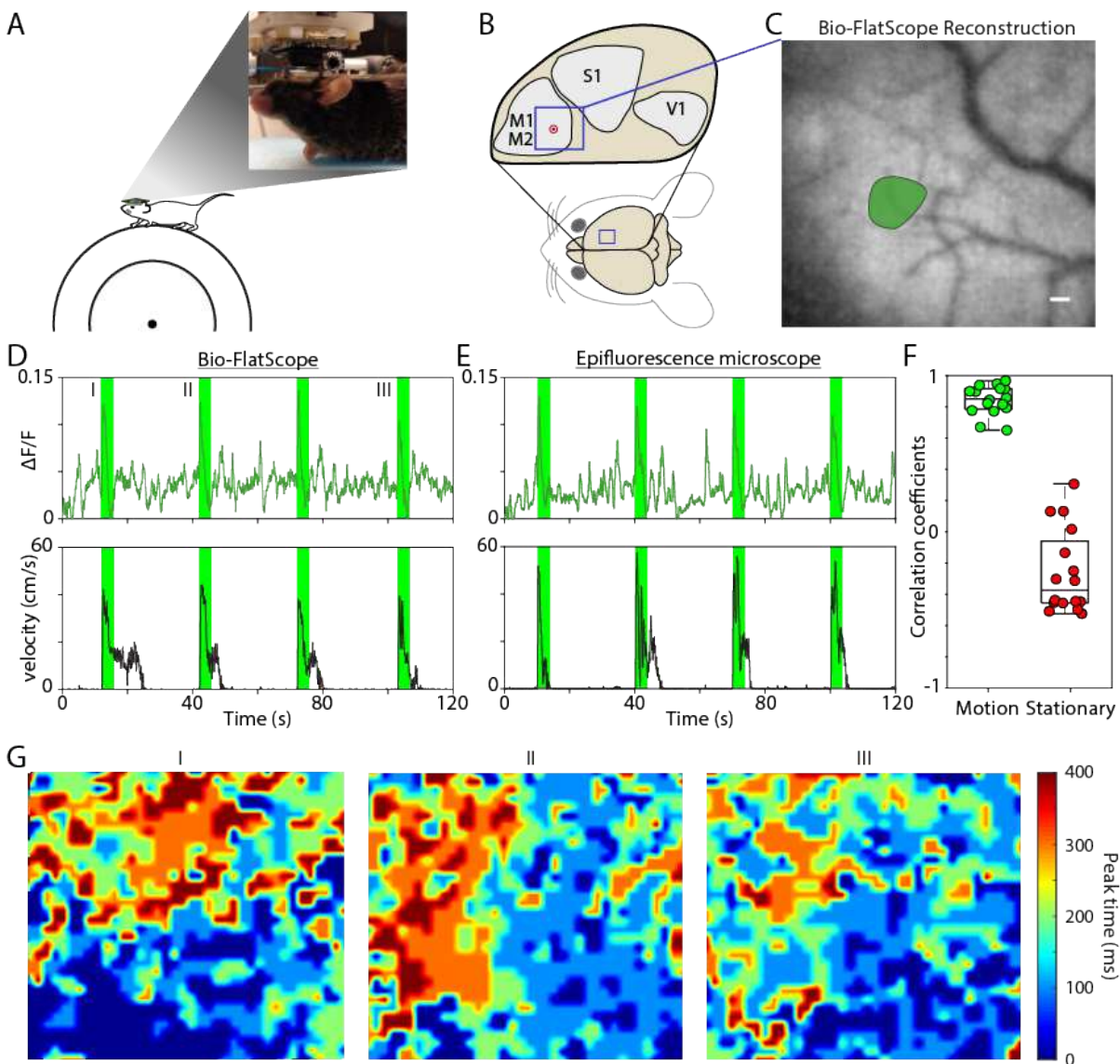

Fig. 3. Comparison of Bio-FlatScope to epifluorescence during stimulus-evoked $\mathrm{Ca}^{2+}$ responses in motor cortex. (A) Experimental setup for Bio-FlatScope for imaging a head-fixed mouse on a freely moving treadmill. Zoom-in shows a photo of a mouse on the treadmill with Bio-FlatScope in place (B) Target region in the mouse brain. The blue square region indicates the approximate location of the cropped FOV (in the motor cortex). The circle with the black dot indicates the approximate region of injection for GCaMP6f. (C) Bio-FlatScope reconstruction with a single ROI of high-activity marked. Scale bar, $100 \mu \mathrm{m}$. (D) $\Delta \mathrm{F} / \mathrm{F}$ trace and treadmill velocity for Bio-FlatScope during recording session. The rising edge of the 4-second windows in green correspond to the application of tactile stimuli. (E) $\Delta \mathrm{F} / \mathrm{F}$ trace and treadmill velocity for epifluorescence during recording session. (F) Box plot showing correlation coefficients comparing stimulus-evoked $\mathrm{Ca}^{2+}$ responses from Bio-FlatScope to epifluorescence (shown in green), and comparison of periods of little or no motion with Bio-FlatScope to stimulus-evoked $\mathrm{Ca}^{2+}$ responses with epifluorescence (shown in red) for the ROI. (G) Heat maps for Bio-FlatScope reconstructions showing spatiotemporal $\mathrm{Ca}^{2+}$ dynamics time-aligned with stimuli (at I, II, and III). Colormap shows the time at which pixels have their peak response for $\Delta \mathrm{F} / \mathrm{F}$ during a $400 \mathrm{~ms}$ period. These data show that when initiating movement, neural activity consistently begins in the lower right and propagates to the upper left.

observed with epifluorescence imaging confirming that Bio-FlatScope measures similar spatiotemporal $\mathrm{Ca}^{2+}$ dynamics (images are processed for visualization, see Fig. S8 for epifluorescence data). The recorded video was captured at $10 \mathrm{~Hz}$ (50 ms exposures) and shows a period of $400 \mathrm{~ms}$ following the tactile stimulation. 

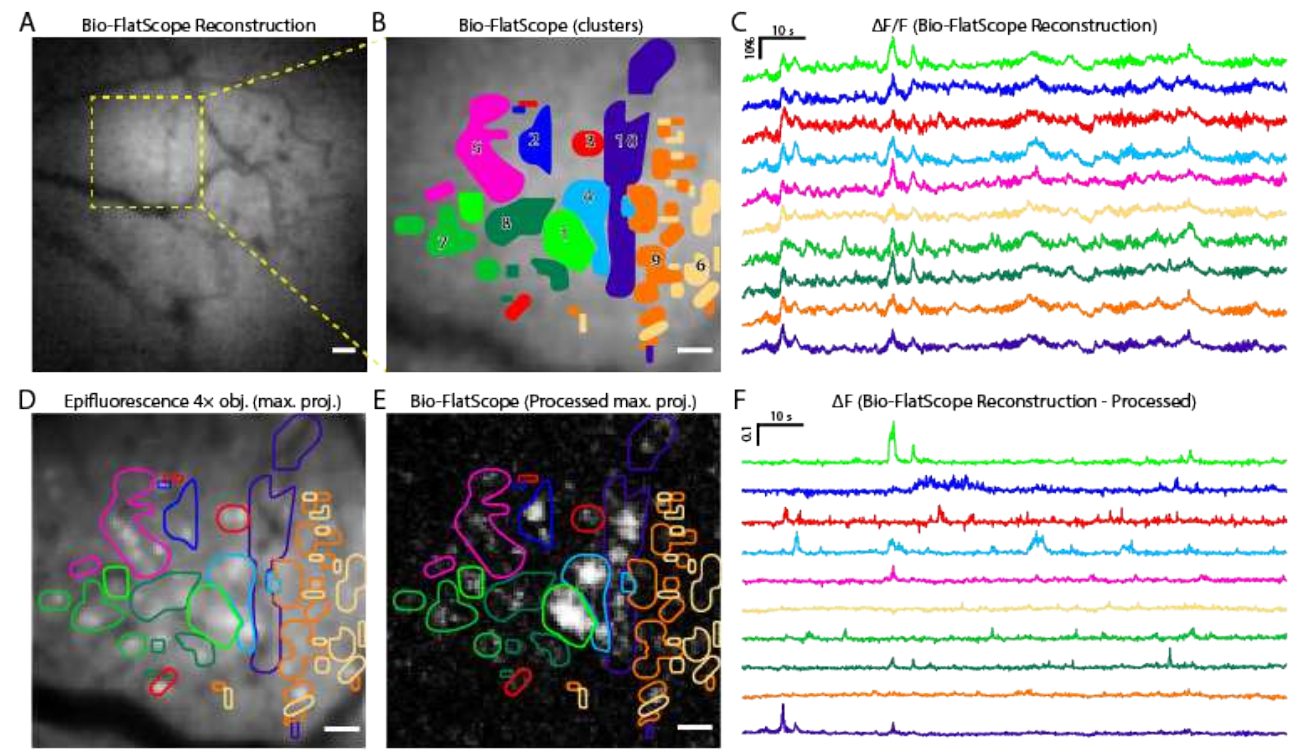

Fig. 4. Extracting near cellular-sized $\mathrm{Ca}^{2+}$ signals from Bio-FlatScope reconstructions. (A) Cropped region of BioFlatScope reconstruction of a single frame with a high-activity region shown by a dashed box. Scale bar, $100 \mu \mathrm{m}$. (B), Zoom-in on the region of high-activity with overlay of clusters determined through post-processing using RPCA and k-means. Scale bar, $50 \mu \mathrm{m}$. (C) $\Delta \mathrm{F} / \mathrm{F}$ traces from Bio-FlatScope reconstruction video over during twominute recording corresponding to the clusters. (D) Maximum projection from epifluorescence recording of same high activity region with overlay of clusters determined from Bio-FlatScope data. Scale bar $50 \mu \mathrm{m}$. (E) Maximum intensity projection of Bio-FlatScope reconstruction data processed using RPCA with overlay of clusters. Scale bar $50 \mu \mathrm{m}$. (F) $\Delta \mathrm{F}$ traces from Bio-FlatScope reconstructions after post-processing using RPCA.

We can also extract unique calcium activity from small regions of interest (ROIs) that approach the size of individual cells. To capture this data, we rely on the fact in the time domain, some $\mathrm{Ca}^{2+}$ activity in individual or small groups of cells appear as outliers compared to the background activity. Robust Principal Component Analysis (RPCA) excels at determining such sparse outliers $[44,45]$, and so we used the RPCA algorithm to identify these regions of high calcium activity. We then used K-means to identify which high-activity regions show correlated activity. These clustered ROIs represent distinct calcium signals from areas that are the size of a few individual cells. When we compared the ROIs determined through RPCA and k-means from Bio-FlatScope we found close correspondence to bright individual neurons observed from epifluorescence microscopy (Fig. 4d). Additionally, the calcium activity in each of these ROIs show unique temporal dynamics suggesting that calcium dynamics might be recovered with near cellular resolution.

It is important to note that the blurring and loss of contrast resulting from scattering affects the properties of the scene but has no direct effect on the performance of our reconstruction algorithm because the reconstruction algorithm makes no assumptions about the medium between the source and the imager. Compared to epifluorescence microscopy, the only additional effect of scattering on lensless imaging is the fact that noise is amplified by the reconstruction algorithm, which is true for all lensless imaging reconstruction techniques, and mitigated by the contour PSF of Bio-FlatScope.

\section{Human oral mucosa imaging}

In addition to pre-clinical imaging, we found that the Bio-FlatScope can image human microvasculature in vivo, which is a clinically relevant biomarker for diseases like sepsis [46, 47], oral neoplasia [48-50], and esophageal neoplasia [51-54]. To test our ability to image microvasculature we imaged oral mucosa in healthy human volunteers. The Bio-FlatScope 
A
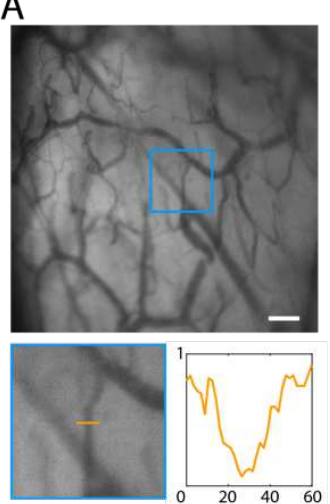

C

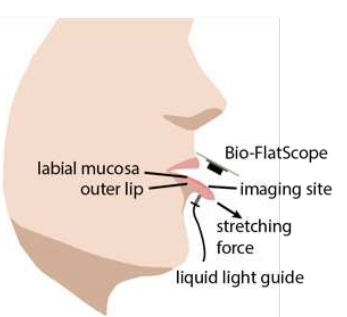

D
B
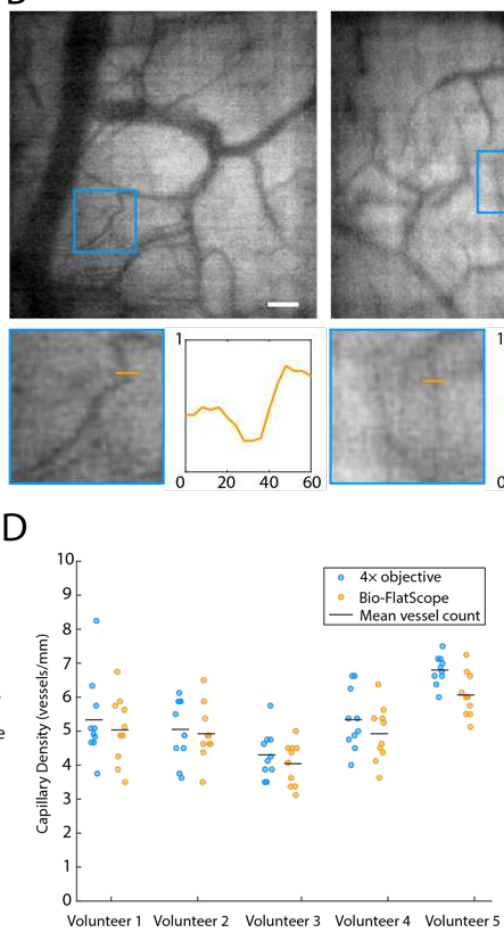
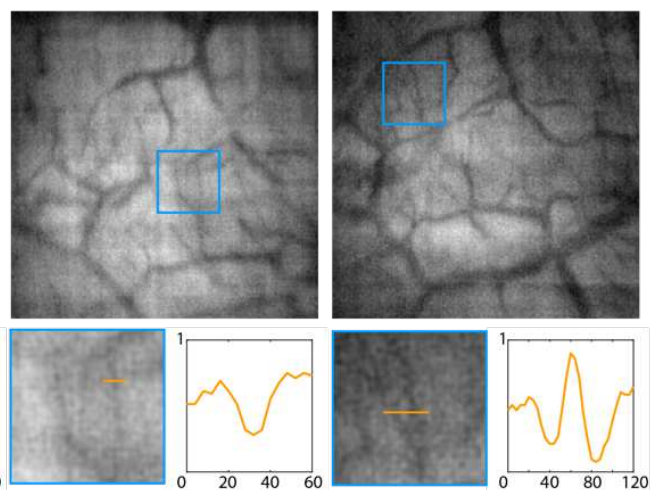

$\mathrm{E}$

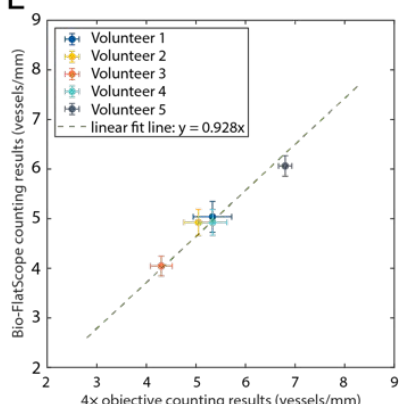

Fig. 5. Human oral mucosa imaging. (A) Images acquired using a $4 \times$ objective. Scale bar, $200 \mu \mathrm{m}$. (B) Reconstructed images from a Bio-FlatScope prototype. Each blue square indicates a sub-region which contains $\sim 15$ $\mu \mathrm{m}$ diameter vessels. Scale bar, $200 \mu \mathrm{m}$. Left insets, zoom-ins of sub-regions. Right insets, pixel intensity along the cross section (shown as orange bars) of the vessels. Length of orange bars in insets, $60 \mu \mathrm{m}$ (120 $\mu \mathrm{m}$ in the 4th inset). X-axis: distance (in $\mu \mathrm{m}$ ) from the left origin of the orange bar. Y-axis: Pixel intensity in normalized arbitrary units, shared by all 4 plots. (C) Experimental setup. The transmissive illumination is delivered by a liquid light guide connected to a green LED light source. (D) Vessel counting results of each volunteer. Each outlined circle corresponds to a different region on the lip. Less translucency indicates overlapping dots. There are 10 circles in each column. (E) Relationship between $4 \times$ objective and Bio-FlatScope counting results. Each circle is the average of 10 different lip regions as shown in panel D. Note that we imaged the same approximate of the labial mucosa with both the $4 \mathrm{X}$ and Bio-FlatScope; however, were unable to capture images of the exact same area of tissue when switching between imaging modalities. The error bars show the standard error of the mean.

prototype was placed a few millimeters away from the inner surface of the lower lip to image the microvasculature in the labial mucosa using transillumination with a green LED coupled to a liquid light guide in contact with the outer surface of the lower lip (Fig. 5). We can see in Fig. $5 \mathrm{a} \& \mathrm{~b}$ that Bio-FlatScope successfully reconstructed the morphological characteristics of microvasculature in labial mucosa with contrast comparable to a $4 \times$ objective, and with the ability to resolve micro vessels less than $20 \mu \mathrm{m}$ in diameter. The measurements in Fig 5d\&e were based on images captured from 10 different nearby regions. Importantly we find that individual differences in microvascular density measured with a $4 \times$ objective lens correlate with those measured using the Bio-FlatScope. For this comparison we captured $4 \times$ and BioFlatScope images from approximately the same region of the oral mucosa; however, because there was no way to accurately register images between recording modalities, all measurements represent slightly different regions of tissue. Changes in microvessel density are important in a number of clinical scenarios: angiogenesis is a hallmark of cancer [55]; microvessel density is correlated with prognosis for a number of cancers [56, 57]; and sub $20-\mu \mathrm{m}$ vessel density has been proposed as a biomarker of sepsis [46,47]. 
The ability to quantify microvascular density combined with the ability to measure vessel morphology suggests that our lensless imaging technology could be used for clinical microvascular imaging with key advantages associated with lensless imaging. Specifically, our small form factor is useful for accessing difficult-to-reach areas of the body and is advantageous for low-resource settings where high resolution in vivo imaging techniques may be prohibitively expensive. This approach enables more patient-friendly form factors and larger fields of view compared to conventional microscopes. In addition, Bio-FlatScope has advantages compared to conventional microscopy where the ability to capture 3D information and to computationally refocus could enable rapid imaging over curved surfaces and provide additional diagnostic information.

\section{Discussion}

Here we have demonstrated structural and functional imaging of biological samples using lensless microscopes with cellular resolution ( $\sim 9$ micron), large FOVs $>16 \mathrm{~mm}^{2}$, digital refocusing, and single capture, three-dimensional imaging over volumes of $4 \mathrm{~mm}^{3}$. The key innovation of a contour PSF enables what is, to the best of our knowledge, the first in vivo $\mathrm{Ca}^{2+}$ imaging and human microvascular imaging with a lensless microscope.

Key next steps will be to improve the packaging and electronic components to make compact devices that could be used for preclinical studies of dynamic calcium activity, endoscopy and superficial biomedical imaging. In the case of $\mathrm{Ca}^{2+}$ imaging we were able to extract localized $\mathrm{Ca}^{2+}$ signals ranging in area from $150 \mu^{2}$ to $0.01 \mathrm{~mm}^{2}$ using RPCA. These results serve as a proof-of-principle that lensless microscopy provides a route toward highresolution and large FOV calcium imaging in a small lightweight form factor. By reducing (or rearranging) the electronic components around the imaging sensor, we foresee miniaturizing our device to the point that we can mount it atop a mouse or small animal to study calcium activity during unrestricted behavior, similar to mini-scopes, but with a superior combination of spatial resolution and field of view.

While the resolution shown here appears to meet the needs for microvascular imaging and approaches cellular resolution $\mathrm{Ca}^{2+}$ imaging, there is still room for improvement. This improvement could be realized by optimizing the masks for closer scene-to-mask distances. These closer distances will not only improve spatial and axial resolution but will increase light collection efficiency and reduce the form-factor enabling easier integration. Additionally, integration of the device can allow for a smaller refractive index change between the sample and device surface, which can cause a slight barrel effect at the edge of the FOV. As one brings the device closer to the sample, there is a corresponding decrease in the FOV until it matches the size of the sensor. To increase the effective FOV, future devices could be arrayed to maintain large scene coverage while maintaining a thin device profile.

Other modifications to the design could further improve performance. Integration of the excitation light source (or multiple sources) can be introduced to improve the illumination coverage over the FOV. For this purpose, commercially available micro-LEDs or integrated waveguides can be used at little to no cost to form factor. To improve on the low-light capabilities necessary for quality fluorescence microscopy, sCMOS imaging sensors or single photon avalanche diodes (SPADs) could be integrated to increase the SNR which is so important in lensless imaging. In addition to physical alterations of the lensless system, integration of new computational methods for imaging through scattering media might be used to extract additional three-dimensional information for brain imaging. One important opportunity for this class of lensless in vivo imagers is the potential to image tens of thousands of neurons simultaneously in a freely moving animal. Additionally, because our system is designed to capture incoherent sources, other imaging modalities like brightfield, darkfield and reflected-light microscopy are also possible, as we demonstrated in the case of microvascular 
imaging without the use of any contrast agents. These lensless microscopy modalities could be useful for endoscopy and point-of-care diagnostics.

Overall, technologies based on Bio-FlatScope can be applied to a number of challenging microscopy problems where high temporal resolution, large FOV, and small form-factor are outside the capabilities of traditional lens-based microscopy.

\section{Methods}

\section{Calibration}

The PSF of the mask must be learned prior to capturing scenes through a one-time calibration process. Because we cannot capture images of an ideal point source, we instead use a single $10 \mu \mathrm{m}$ fluorescent microsphere (FluoSpheres yellow-green), which is closely aligned to the center of our mask. The fluorescent bead was dropcast onto a microscope slide, then protected by a coverslip (Nexterion, $170 \mu \mathrm{m}$ thick). Images of the PSF were captured for each depth (i.e., distance axially from the device) of interest by Bio-FlatScope with the bead located approximately at the center of the phase mask. Calibration images were averaged through multiple captures and background was subtracted to ensure the highest SNR for the PSFs. The sensitivity of the system to depth requires calibration images of PSFs to be taken over a range of distances, which allow for refocusing and three-dimensional reconstruction in postprocessing.

\section{Reconstruction}

To reconstruct images, we effectively solve the following minimization problem, adding Tikhonov regularization to the deconvolution to avoid noise amplification:

$$
\hat{\mathbf{i}}=\arg \min _{\mathbf{i}}\|\mathbf{b}-p * \mathbf{i}\|_{F}^{2}+\frac{\gamma}{2}\|\mathbf{i}\|_{2}^{2}
$$

where $*$ denotes convolution, $\hat{\imath}$ is the estimate of the scene, $\mathbf{i}$ is the scene, $\mathbf{b}$ is the sensor measurement, $p$ is the PSF at the scene distance, $\gamma$ is the regularization weight, and $\mathrm{F}$ is the Frobenius norm. This minimization problem can be solved by closed form via Wiener deconvolution as:

$$
\hat{\mathbf{i}}=\mathcal{F}^{-1}\left(\frac{\mathcal{F}(p)^{*} \odot \mathcal{F}(\mathbf{b})}{|\mathcal{F}(p)|^{2}+\gamma}\right)
$$

where $\odot$ denotes Hadamard product, $F$ denotes a Fourier transform and $F^{-1}$ denotes an inverse Fourier transform. To reconstruct three-dimensionally, we solve the same minimization problem, but do so for the sum of distances of interest:

$$
\hat{\mathbf{i}}_{D}=\arg \min _{\mathbf{i}_{D}}\left\|\mathbf{b}-\sum_{d=1}^{D} p_{d} * \mathbf{i}_{d}\right\|_{2}^{2}+\frac{\gamma}{2}\left\|\mathbf{i}_{D}\right\|_{1}
$$

where $D$ is the number of distances, $d$, from the imaging device.

\section{Excitation Light}

Excitation light for Bio-FlatScope was achieved both transmissively and in near-epi configurations. Transmissive illumination was provided either using light through an epifluorescent microscope objective (Nikon Fluor $4 \times$, NA $0.13 \mathrm{PhL}$ DL), or using a nearcollimated $470 \mathrm{~nm}$ LED (Thorlabs, M470L3) with an incorporated GFP excitation filter (Thorlabs, MF469-35). Near-epi illumination was provided using a fiber-coupled $475 \mathrm{~nm}$ LED (Prizmatix, UHP-T-475-SR) with an incorporated GFP excitation filter (Thorlabs, MF469-35). The light was coupled into a multimode fiberoptic cable (Thorlabs M72L01 $200 \mu \mathrm{m}$ diameter core, NA 0.39 or Edmund Optics, \#57-749 $400 \mu$ m diameter core NA 0.22) with an aluminum coated microprism (Edmund Optics $0.18 \mathrm{~mm} \# 66-768$ or $0.70 \mathrm{~mm} \# 66-773$ ) adhered to the 
exposed tip of the fiber with optical epoxy (Norland, NOA72). The microprism was placed near the surface of the lensless imaging device.

\section{Fabrication}

Bio-FlatScope prototypes were constructed using an off-the-shelf board level camera (Imaging Source, DMM 37UX178-ML) with a monochrome Sony CMOS imaging sensor (IMX178LLJ, 6.3 MP, $2.4 \mu \mathrm{m}$ pixels). The phase mask was fabricated using a 3-D photolithographic system (Nanoscribe, Photonic Professional GT2) using a photoresist (Nanoscribe, IP-L 780) on a 700 $\mu \mathrm{m} \mathrm{SiO} 2$ substrate. The substrate was then cut down to more closely match the sensor size of the camera (and filters). The substrate was masked with an opaque material to provide an aperture containing only the phase elements. The substrate with phase mask was affixed atop the imaging sensor, followed by a hybrid filter, similar to Richard et al. [58], but using a commercially available absorptive filter (Kodak, Wratten \#12) placed below an interference filter (Chroma, ET525/50m), both cut to the sensor size requirements (see Fig. 1a). A housing was 3D printed (MJP 2500) to hold the phase mask and filters atop the imaging sensor.

\section{Mouse Brain Slice}

All experiments were approved by the Rice University IACUC. Mice were sacrificed 14 days post-injection of AAV9-CamKII-GCaMP6f in the CA1 region of the hippocampus. From bregma, the injection site was $+2 \mathrm{~mm}$ medial/lateral, $-2 \mathrm{~mm}$ anterior/posterior and $-1.65 \mathrm{~mm}$ deep. The total injection amount was $0.5 \mu \mathrm{L}$ at a rate of $0.05 \mu \mathrm{L} / \mathrm{min}$. The brain expressing GCaMP6f was cryoprotected in 30\% sucrose, embedded in OCT compound (Tissue-Tek), frozen and sliced into $50 \mu \mathrm{m}$ slices using a cryostat (Leica). Slices were then rinsed in $1 \mathrm{x}$ phosphate-buffered saline (PBS) and mounted using Vectashield H-1000 with DAPI (Vector Labs) then sealed with coverslips.

\section{Hydra vulgaris}

Hydra were cultured in Hydra media using the protocol adapted from Steele Lab (UC Irvine) at $18^{\circ} \mathrm{C}$ with $12: 12$ hours light-dark cycle. Animals were fed freshly hatched artemia every 2 days and cleaned after 4 hours. Transgenic lines developed by embryo microinjections [28] expressing either GFP in interstitial cell lineage (GFP, Neurons) or GCaMP7b in endodermal muscle cells (GCaMP7b, Endo) were used. The transgenic line of Hydra expressing GFP under actin promoter was originally developed by Steele Lab and selected for expression in neurons. The transgenic line of Hydra expressing GCaMP7b under EF1a promoter was developed by Robinson Lab and Juliano lab (UC Davis) selected for expression in endodermal muscle cells.

Both Hydra expressing GFP and GCaMP7b were imaged in hydra media on petri dishes with coverslip bottoms (MatTek, $35 \mathrm{~mm}, 14 \mathrm{~mm}$ \#1.5, coverslip). Bio-FlatScope images were captured through the coverslip with excitation light provided by the fiberoptic cable/microprism combination.

\section{Brain tissue phantom}

The brain tissue phantom was created by suspending non-fluorescent polystyrene microspheres (Polybead Acrylate Microspheres, $1 \mu \mathrm{m}$ diameter, $4.55 \times 10^{10}$ beads $/ \mathrm{mL}$ ) in polydimethylsiloxane (PDMS) (Sylgard, Dow Corning; 10:1 elastomer:cross-linker weight ratio). For the brain tissue phantom, a concentration of $5.46 \times 10^{9}$ beads $/ \mathrm{mL}$ was used to achieve a scattering coefficient of $\sim 1 \mathrm{~mm}^{-1}$ to simulate the reported scattering coefficients in mouse brain tissue [60,61]. Microspheres $(0.6 \mathrm{~mL})$ were added to isopropyl alcohol $(0.6 \mathrm{~mL})$ and vortexed thoroughly for uniformity. The microsphere/alcohol mixture was added to $4 \mathrm{~g}$ of the PDMS elastomer and mixed thoroughly, followed by $0.4 \mathrm{~g}$ of crosslinker and again mixed thoroughly for uniformity. The desired thickness of $140 \mu \mathrm{m}$ was achieved by spin-coating the mixture onto a $\mathrm{SiO}_{2}$ wafer. then heated at $37^{\circ} \mathrm{C}$ for 30 minutes. 
For fluorescence imaging we illuminated the sample with a fiberoptic cable attached to an aluminum-coated microprism [61]. This illumination strategy is capable of fitting into the small space between the lensless imager and biological sample. To prevent the excitation light from reaching the sensor and overwhelming the fluorescence signal we constructed a hybrid excitation filter that combines an absorptive and interference filter [58] and attached it to the top of the phase mask. The thickness between the image sensor and surface of device is $\sim 5 \mathrm{~mm}$.

\section{Animals for in vivo mouse brain imaging}

Wild-type C57BL/6 mice ( $\mathrm{n}=3$ ) from Charles River Laboratories were used for this study. Animals are housed with standard $12 \mathrm{~h}$ light/dark cycle with ad libitum food and water. All animals $(\mathrm{n}=3)$ were injected with the adeno-associated viral vector AAV9.CamKII.GCaMP6f.WPRE.SV40 (Penn Vector Core). All experimental procedures were approved by the Institutional Animal Care and Use Committee at Rice University and followed the guidelines of the National Institute of Health.

\section{Headpost Implant and Cranial Window Design}

Headpost implant design was adapted from Ghanbari et al. [62], and consists of a custom-made Titanium or Stainless-steel head-plate, a 3D-printed frame, and three 0-80 screws to hold the frame to the head-plate. We fabricated the head-plate with Titanium or Stainless-steel plate (McMaster-Carr) using a waterjet system (OMax), and 3D printed the frame using a ProJet MJP 2500 (3D Systems). Our design files can be found online (https://github.com/ckemere/TreadmillTracker/tree/master/UMinnHeadposts). We assembled the headpost implant after tapping the 3D printed frame with 0-80 tap and securing the headplate over the frame with three screws. The entire headpost is then stored in $70 \%$ Ethanol prior to surgery.

Cranial window fabrication procedure was adapted from Goldey et al. [63]. Windows were made of 2 stacked round coverslips (Warner Instruments \# CS-3R, CS-4R, CS-5R) of different diameters. To fabricate the stacked windows, a $3 \mathrm{~mm}$ (or $4 \mathrm{~mm}$ ) round coverslip was epoxied to a $4 \mathrm{~mm}$ (or $5 \mathrm{~mm}$ ) cover slip using an optical adhesive (Norland Products Inc. e.g. \# NOA $61,71,84$ ) and cured using long-wavelength UV light. To accommodate the large $5 \mathrm{~mm}$ stacked window, we cut off the right side of the 3D-printed frame to allow for extra space for the C\&B Metabond to bind to the skull outside of the stacked cranial window. Fabricated stacked windows were stored in $70 \%$ ethanol prior to surgery.

\section{Surgical Procedures}

For AAV9 injections, mice were anesthetized with $1-2 \%$ isoflurane gas in oxygen and administered sustained release Buprenorphine SR-LAB (Zoofarm, $0.5 \mathrm{mg} / \mathrm{kg}$ ). A small craniotomy was carefully drilled at the target location. A total of $0.5-1 \mu \mathrm{L}$ of AAV9 virus was injected slowly at a rate of $0.07 \mu \mathrm{L} / \mathrm{min}$ into each mouse with a Hamilton syringe paired with syringe pump controller (KD Scientific \# 78-0311). Specifically, mouse W1 was injected at 1.5 AP; +1.5 ML; -0.25 DV targeting the motor cortex; mouse W2 was injected at -1.67 AP; $+1.1 \mathrm{ML} ;-0.25 \mathrm{DV}$ targeting the motor cortex; mouse W3 was injected at -1.23 AP; +1.23 ML; $-0.25 \mathrm{DV}$ targeting the motor cortex. Following the injection, a small amount of bone wax was applied over the craniotomy, while taking care not to press down on the brain surface. The incisions were closed using a small drop of Vetbond (3M), and the mice were allowed to express for at least 4 weeks before headpost and window implantation.

For headpost and window implantation, mice were administered Buprenorphine SR-LAB $(0.5 \mathrm{mg} / \mathrm{kg})$ and Dexamethasone $(2 \mathrm{mg} / \mathrm{kg}) 30 \mathrm{~min}$ prior to the craniotomy procedure. Mice were anesthetized with 1-2.5\% of isoflurane gas in oxygen and secured on a stereotax (Kopf Instruments) using ear bars. A single large cut was made to cut away the majority of the skin 
above the skull while attempting to match the 3D printed frame to the exposed skull. We then used a $3 \mathrm{~mm}$ (or $4 \mathrm{~mm}$, depending on the window implant size) biopsy punch to carefully center the craniotomy over the AAV injection site while avoiding the sagittal suture, and slowly and gently rotated the biopsy punch until the bone could be lifted away with 5/45 forceps. We used saline to irrigate regularly while performing the craniotomy, taking care not to puncture the dura. After stopping any bleeding, we placed a small amount of silicone oil (Sigma-Aldrich Inc. \# 181838) to cover the brain, then carefully placed the stacked window over the brain. We then applied pressure to the top of the stacked window with a thinned wooden applicator (e.g. back of a cotton swab or toothpick) mounted on the stereotaxic arm until the $4 \mathrm{~mm}$ (or $5 \mathrm{~mm}$ ) coverslip was flush with the skull surface. We used cyanoacrylate to glue around the window and waited until it dried before removing pressure from the thinned wooden applicator. We then positioned the headpost over the skull and used C\&B Metabond to cement the headpost in place, taking care not to cover the cranial window. After the Metabond dried, we applied some silicone elastomer (World Precision Instruments, Kwik-Sil) over the cranial window to protect it from any damage. We then administered post-operative drugs (meloxicam at $5 \mathrm{mg} / \mathrm{kg}$ and $0.25 \%$ Bupivicaine around the headpost implant) and allowed the mouse to recover for at least 3 days before imaging.

\section{Recording sessions}

Prior to each animal's first awake head-fixed imaging session, the animals were acclimated to head-fixation and the treadmill set-up for at least three sessions. Additionally, to minimize stress prior to awake imaging session, we acclimated the animals to mechanical fixation in which the head was restrained. Animals received chocolate milk (Nesquik) as a reward while running on a custom-built treadmill. Once acclimated, animals were imaged for up to two sessions per day, where each session lasted no longer than two hours.

For experiments, mice were head-fixed atop a freely moving treadmill during video capture. Epifluorescence microscope images were captured with a sCMOS camera (Kiralux, 5 MP) through a $4 \times$ objective. Treadmill rotation data (synchronized with the camera) was captured during the experiment. During the recording session, a brushing tactile stimulus was applied to the spine region of the mouse with a minimum of $30 \mathrm{~s}$ between stimulus events. Bio-FlatScope recording sessions were captured within 30 minutes of epifluorescence recordings, with exposure time, recording lengths and stimuli timing matching those with the epifluorescence microscope. ROIs in the brain for comparing treadmill activity across epifluorescence and BioFlatScope were selected based on high activity areas extracted from the epifluorescence video.

\section{RPCA analysis}

This analysis was based on two-minute imaging sessions recorded at $20 \mathrm{~Hz}$ over the full FOV of the cranial window using both Bio-FlatScope and an epifluorescence microscope with a $4 \times$ objective. The ROIs for cluster analysis were selected by observing a $500 \mu \mathrm{m}$ x $500 \mu \mathrm{m}$ region of spontaneous high activity in the Bio-FlatScope reconstructions (Fig. 4a). This same ROI was used for both Bio-FlatScope and epifluorescence data. Figures $4 \mathrm{~b} \& \mathrm{c}$ show the clusters extracted through $\mathrm{k}$-means as well as the corresponding $\Delta \mathrm{F} / \mathrm{F}$ for those clusters.

\section{Human subjects for oral mucosa imaging}

The in vivo human study was performed at Rice University in Houston, TX. Volunteers recruited in the study were 18 years or older with no underlying health conditions. All volunteers provided health pre-screen information to reduce the risk of COVID spread. The study was in accord with the protocol approved by the Institutional Review Board at Rice University. Written informed consent was obtained from all volunteers prior to imaging.

\section{In vivo oral mucosa imaging}


Bio-FlatScope imaging and microscopic imaging were performed on each human volunteer. A Bio-FlatScope prototype based on a contour phase mask with $6 \mu \mathrm{m}$ feature size was used in this experiment. During a Bio-FlatScope imaging session, volunteers were instructed to place their chin and forehead on a custom chin-head rest for maximum stability (Fig. S13). The illumination was achieved by a tabletop green LED (X-Cite XLED1) and a liquid light guide ( $3 \mathrm{~mm}$ diameter) mounted in the chin rest with illumination intensity less than $20 \mathrm{~mW}$. The illumination source was used 3 to 5 minutes per imaging session, resulting in maximum illumination intensity at skin less than the threshold limit value (TLV) specified by ACGIH. During the experiment, the volunteer positioned his/her lower lip over the tip of the liquid light guide with one hand and placed the imaging device onto the illuminated area with the other hand. The imaging device consists of a Bio-FlatScope prototype and an opaque handheld enclosure, designed to maintain a constant $3 \mathrm{~mm}$ working distance, with a $2.5 \mathrm{~mm}$ wide square aperture.

The microscopic imaging session was performed with a microscope objective (Nikon Fluor $4 \times$, NA 0.13 PhL DL). During the imaging session, the volunteer was instructed to position his/her lower lip with one hand and hold the liquid light guide with the other hand to keep the tip in contact with the bottom side of the lower lip.

\section{Human oral mucosa vessel counting}

Vessel counting was based on images taken by the $4 \times$ objective, and reconstructed images from the Bio-FlatScope prototype. A grid containing $2 \mathrm{~mm}$ of horizontal lines and $2 \mathrm{~mm}$ of vertical lines was superimposed on the image. The capillary density was calculated similar to the method in $[46,47]$. The number of small vessels $(<20 \mu \mathrm{m})$ per millimeter was calculated as the number of small vessels crossing the grid divided by the total length of the grid. Two researchers independently performed vessel counting for each image and the results were averaged. In each volunteer, the images from ten different areas were averaged to give a final capillary density value.

\section{Funding, acknowledgements, and disclosures}

\section{Funding}

This work was supported in part by DARPA grant N66001-17-C-4012.

\section{Acknowledgements}

We would like to thank Krishna Badhiwala for the useful discussions on Hydra vulgaris neurobiology and behavior. We would also like to thank Jill Juneau for the mounted mouse brain slices.

\section{Data and code}

The data and code that support the findings of this study are available from the authors upon reasonable request.

\section{See Supplementary Information for supporting content}


505 1. J. Holloway, Y. Wu, M. K. Sharma, O. Cossairt, and A. Veeraraghavan, "SAVI: Synthetic apertures for longrange, subdiffraction-limited visible imaging using Fourier ptychography," Sci. Adv. 3, e1602564 (2017).

2. Y. Wu, M. K. Sharma, and A. Veeraraghavan, "WISH: wavefront imaging sensor with high resolution," Light Sci. Appl. 8, 44 (2019).

3. V. Boominathan, J. Adams, J. Robinson, and A. Veeraraghavan, "PhlatCam: Designed phase-mask based thin lensless camera," IEEE Trans. Pattern Anal. Mach. Intell. 1-1 (2020).

4. E. Betzig, G. H. Patterson, R. Sougrat, O. W. Lindwasser, S. Olenych, J. S. Bonifacino, M. W. Davidson, J. Lippincott-Schwartz, and H. F. Hess, "Imaging intracellular fluorescent proteins at nanometer resolution.," Science 313, 1642-5 (2006).

5. M. J. Rust, M. Bates, and X. Zhuang, "Sub-diffraction-limit imaging by stochastic optical reconstruction microscopy (STORM)," Nat. Methods 3, 793-796 (2006).

6. L. Tian and L. Waller, "3D intensity and phase imaging from light field measurements in an LED array microscope," Optica 2, 104 (2015).

7. R. Prevedel, Y.-G. Yoon, M. Hoffmann, N. Pak, G. Wetzstein, S. Kato, T. Schrödel, R. Raskar, M. Zimmer, E. S. Boyden, and A. Vaziri, "Simultaneous whole-animal 3D imaging of neuronal activity using light-field microscopy," Nat Methods 11, 727-730 (2014).

8. T. S. Ralston, D. L. Marks, P. Scott Carney, and S. A. Boppart, "Interferometric synthetic aperture microscopy," Nat Phys 3, 129-134 (2007).

9. A. Ozcan and U. Demirci, "Ultra wide-field lens-free monitoring of cells on-chip.," Lab. Chip 8, 98-106 (2008)

10. J. K. Adams, V. Boominathan, B. W. Avants, D. G. Vercosa, F. Ye, R. G. Baraniuk, J. T. Robinson, and A. Veeraraghavan, "Single-frame 3D fluorescence microscopy with ultraminiature lensless FlatScope," Sci. Adv. 3, e1701548 (2017)

11. N. Antipa, G. Kuo, R. Heckel, B. Mildenhall, E. Bostan, R. Ng, and L. Waller, "DiffuserCam: lensless singleexposure 3D imaging," Optica 5, 1 (2018).

12. A. Greenbaum, W. Luo, T.-W. Su, Z. Göröcs, L. Xue, S. O. Isikman, A. F. Coskun, O. Mudanyali, and A. Ozcan, "Imaging without lenses: achievements and remaining challenges of wide-field on-chip microscopy," Nat. Methods 9, 889-895 (2012).

13. P. R. Gill and D. G. Stork, "Lensless Ultra-Miniature Imagers Using Odd-Symmetry Spiral Phase Gratings," Imaging Appl. Opt. CW4C.3 (2013).

14. S. Asif, A. Ayremlou, A. Sankaranarayanan, A. Veeraraghavan, and R. Baraniuk, "FlatCam: Thin, Lensless Cameras using Coded Aperture and Computation," IEEE Trans. Comput. Imaging 1 (2016).

15. V. Boominathan, J. K. Adams, M. S. Asif, B. W. Avants, J. T. Robinson, R. G. Baraniuk, A. C. Sankaranarayanan, and A. Veeraraghavan, "Lensless Imaging: A computational renaissance," IEEE Signal Process. Mag. 33, 23-35 (2016).

16. R. Horisaki, Y. Ogura, M. Aino, and J. Tanida, "Single-shot phase imaging with a coded aperture," Opt Lett 39, 6466-6469 (2014).

17. M. J. DeWeert and B. P. Farm, "Lensless coded aperture imaging with separable doubly Toeplitz masks," Opt. Eng. 54, 023102 (2015).

18. G. Kuo, N. Antipa, R. Ng, and L. Waller, "DiffuserCam: Diffuser-Based Lensless Cameras," in Imaging and Applied Optics 2017 (3D, AIO, COSI, IS, MATH, PcAOP) (2017), p. CTu3B.2.

19. I. Sencan, A. F. Coskun, U. Sikora, and A. Ozcan, "Spectral demultiplexing in holographic and fluorescent on-chip microscopy.," Sci. Rep. 4, 3760 (2014).

20. A. F. Coskun, I. Sencan, T.-W. Su, and A. Ozcan, "Lensless wide-field fluorescent imaging on a chip using compressive decoding of sparse objects." Opt. Express 18, 10510-23 (2010).

21. S. R. P. Pavani, M. A. Thompson, J. S. Biteen, S. J. Lord, N. Liu, R. J. Twieg, R. Piestun, and W. E. Moerner, "Three-dimensional, single-molecule fluorescence imaging beyond the diffraction limit by using a double-helix point spread function," Proc Natl Acad Sci U A 106, 2995-2999 (2009).

22. J. Chen, M. Hirsch, R. Heintzmann, B. Eberhardt, and H. P. A. Lensch, "A Phase-coded Aperture Camera with Programmable Optics," Electron. Imaging 2017, 70-75 (2017).

23. Y. Wu, V. Boominathan, H. Chen, and Others, "PhaseCam3D—Learning Phase Masks for Passive Single View Depth Estimation," 2019 IEEE (2019).

24. J. Chang, V. Sitzmann, X. Dun, W. Heidrich, and G. Wetzstein, "Hybrid optical-electronic convolutional neural networks with optimized diffractive optics for image classification," Sci Rep 8, 12324 (2018).

25. W. Chi and N. George, "Optical imaging with phase-coded aperture," Opt Express 19, 4294 (2011)

26. W. Wang, F. Ye, H. Shen, N. A. Moringo, C. Dutta, J. T. Robinson, and C. F. Landes, "Generalized method to design phase masks for 3D super-resolution microscopy," Opt Express 27, 3799-3816 (2019).

27. K. N. Badhiwala, D. L. Gonzales, D. G. Vercosa, B. W. Avants, and J. T. Robinson, "Microfluidics for electrophysiology, imaging, and behavioral analysis of Hydra," Lab Chip 18, 2523-2539 (2018).

28. C. Dupre and R. Yuste, "Non-overlapping Neural Networks in Hydra vulgaris," Curr Biol 27, 1085-1097 (2017).

29. C. E. Juliano, H. Lin, and R. E. Steele, "Generation of transgenic Hydra by embryo microinjection.," J. Vis. Exp. JoVE 51888 (2014). 
30. Badhiwala, K. N., Primack, A. S., Juliano, C. E. \& Robinson, J. T. Multiple nerve rings coordinate Hydra mechanosensory behavior. 1-28 (2020).

31. J. R. Szymanski and R. Yuste, "Mapping the whole-body muscle activity of Hydra vulgaris," Curr Biol (2019)

32. C. N. Tzouanas, S. Kim, K. N. Badhiwala, B. W. Avants, and J. T. Robinson, "Thermal stimulation temperature is encoded as a firing rate in a Hydra nerve ring," bioRxiv 787648 (2019).

33. D. A. Dombeck, M. S. Graziano, and D. W. Tank, "Functional Clustering of Neurons in Motor Cortex Determined by Cellular Resolution Imaging in Awake Behaving Mice," J. Neurosci. 29, 13751-13760 (2009).

34. R. Prevedel, A. J. Verhoef, A. J. Pern'’1a-Andrade, S. Weisenburger, B. S. Huang, T. Nöbauer, A. Fernández, J. E. Delcour, P. Golshani, A. Baltuska, and A. Vaziri, Fast Volumetric Calcium Imaging across Multiple Cortical Layers Using Sculpted Light, No. 12 (2016), Vol. 13.

35. N. J. Sofroniew, D. Flickinger, J. King, and K. Svoboda, "A large field of view two-photon mesoscope with subcellular resolution for in vivo imaging," Elife 5, (2016).

36. D. A. Dombeck, A. N. Khabbaz, F. Collman, T. L. Adelman, and D. W. Tank, "Imaging large-scale neural activity with cellular resolution in awake, mobile mice," Neuron 56, 43-57 (2007).

37. R. Tomer, M. Lovett-Barron, I. Kauvar, A. Andalman, V. M. Burns, S. Sankaran, L. Grosenick, M. Broxton, S. Yang, and K. Deisseroth, "SPED Light Sheet Microscopy: Fast Mapping of Biological System Structure and Function," Cell 163, 1796-1806 (2015)

38. K. K. Ghosh, L. D. Burns, E. D. Cocker, A. Nimmerjahn, Y. Ziv, A. E. Gamal, and M. J. Schnitzer, "Miniaturized integration of a fluorescence microscope," Nat Methods 8, 871-878 (2011).

39. M. P. Vanni, A. W. Chan, M. Balbi, G. Silasi, and T. H. Murphy, "Mesoscale Mapping of Mouse Cortex Reveals Frequency-Dependent Cycling between Distinct Macroscale Functional Modules," J. Neurosci. 37, 7513-7533 (2017).

40. Rynes, M. et al. Miniaturized head-mounted device for whole cortex mesoscale imaging in freely behaving mice. (2020). doi:10.1101/2020.05.25.114892

41. D. Barson, A. S. Hamodi, X. Shen, G. Lur, R. T. Constable, J. A. Cardin, M. C. Crair, and M. J. Higley, "Simultaneous mesoscopic and two-photon imaging of neuronal activity in cortical circuits," Nat. Methods 17, 107-113 (2020).

42. E. A. Pnevmatikakis and A. Giovannucci, "NoRMCorre: An online algorithm for piecewise rigid motion correction of calcium imaging data," J. Neurosci. Methods 291, 83-94 (2017).

43. A. Samaniego, V. Boominathan, A. Sabharwal, and A. Veeraraghavan, "mobileVision," in Proceedings of the Wireless Health 2014 on National Institutes of Health - WH '14 (ACM Press, 2014), pp. 1-8.

44. E. J. Candes, X. Li, Y. Ma, and J. Wright, "Robust Principal Component Analysis?," J. ACM 58, (2011).

45. Z. Lin, A. Ganesh, J. Wright, and L. Wu, "Fast convex optimization algorithms for exact recovery of a corrupted low-rank matrix," Comput. Adv. Ldots 1-18 (2009).

46. Trzeciak, S. et al. Early microcirculatory perfusion derangements in patients with severe sepsis and septic shock: Relationship to hemodynamics, oxygen transport, and survival. Annals of Emergency Medicine 49, (2007).

47. De Backer, D. et al. Microcirculatory alterations in patients with severe sepsis: Impact of time of assessment and relationship with outcome. Critical Care Medicine 41, 791-799 (2013).

48. Takano, J. H. et al. Detecting early oral cancer: narrowband imaging system observation of the oral mucosa microvasculature. International Journal of Oral and Maxillofacial Surgery 39, 208-213 (2010).

49. Yang, S. W. et al. Clinical characteristics of narrow-band imaging of oral erythroplakia and its correlation with pathology. BMC Cancer 15, (2015).

50. Bastos, P. \& Cook, R. Real Time Optical Vascular Imaging: A Potential Technique for the Diagnosis of Mucosal Disease Including Early Oral Cancer. Primary dental journal 5, 86-91 (2016).

51. Ide, E., Maluf-Filho, F., Chaves, D. M., Matuguma, S. E. \& Sakai, P. Narrow-band imaging without magnifcation for detecting early esophageal squamous cell carcinoma. World Journal of Gastroenterology 17, 4408-4413 (2011).

52. Tan, N. C. W., Herd, M. K., Brennan, P. A. \& Puxeddu, R. The role of narrow band imaging in early detection of head and neck cancer. British Journal of Oral and Maxillofacial Surgery 50, 132-136 (2012).

53. Ide, E. et al. Endoscopic detection of early esophageal squamous cell carcinoma in patients with achalasia: Narrow-band imaging versus Lugol's staining. Journal of Oncology (2013). doi:10.1155/2013/736756

54. Sharma, P. et al. Development and Validation of a Classification System to Identify High-Grade Dysplasia and Esophageal Adenocarcinoma in Barrett's Esophagus Using Narrow-Band Imaging. Gastroenterology 150, 591-598 (2016).

55. Hanahan, D. \& Weinberg, R. A. Hallmarks of cancer: The next generation. Cell 144, 646-674 (2011)

56. Wang, J. Z. et al. Clinicopathological and prognostic significance of blood microvessel density in endometrial cancer: a meta-analysis and subgroup analysis. Archives of Gynecology and Obstetrics 297, 731-740 (2018).

57. Ma, G. et al. Microvessel density as a prognostic factor in esophageal squamous cell cancer patients. Medicine (United States) 96, (2017).

58. C. Richard, A. Renaudin, V. Aimez, and P. G. Charette, "An integrated hybrid interference and absorption filter for fluorescence detection in lab-on-a-chip devices.," Lab. Chip 9, 1371-1376 (2009). 
59. M. B. Bouchard, V. Voleti, C. S. Mendes, C. Lacefield, W. B. Grueber, R. S. Mann, R. M. Bruno, and E. M. C. Hillman, "Swept confocally-aligned planar excitation (SCAPE) microscopy for high-speed volumetric imaging of behaving organisms," Nat. Photonics 9, 113-119 (2015).

60. F. Ye, B. W. Avants, A. Veeraraghavan, and J. T. Robinson, "Integrated light-sheet illumination using metallic slit microlenses," Opt. Express 26, 27326-27338 (2018).

61. D. Miyamoto and M. Murayama, "The fiber-optic imaging and manipulation of neural activity during animal behavior," Neurosci Res 103, 1-9 (2016).

62. L. Ghanbari, R. E. Carter, M. L. Rynes, J. Dominguez, G. Chen, A. Naik, J. Hu, M. A. K. Sagar, L. Haltom, N. Mossazghi, M. M. Gray, S. L. West, K. W. Eliceiri, T. J. Ebner, and S. B. Kodandaramaiah, "Cortex-wide neural interfacing via transparent polymer skulls," Nat Commun 10, 1500 (2019).

63. G. J. Goldey, D. K. Roumis, L. L. Glickfeld, A. M. Kerlin, R. C. Reid, V. Bonin, D. P. Schafer, and M. L. Andermann, "Removable cranial windows for long-term imaging in awake mice," Nat Protoc 9, 2515-2538 (2014). 


\section{In vivo imaging with a flat, lensless microscope: 644 supplemental document}

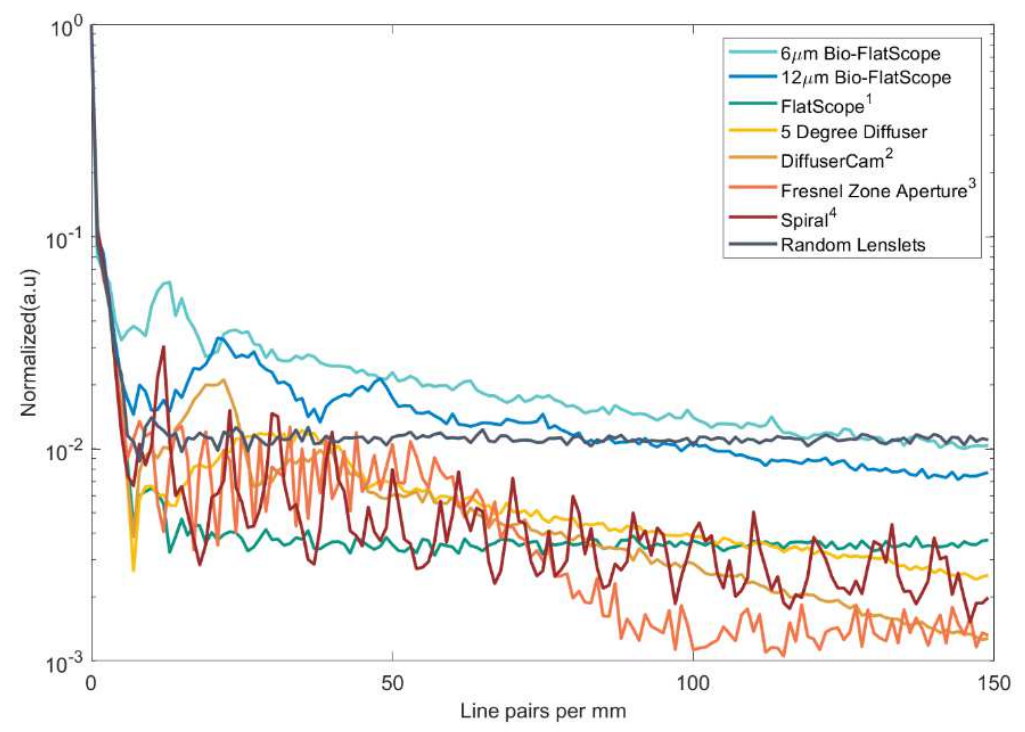

Fig. S1. Modulation transfer function (MTF) comparison among PSF designs used by lensless imaging systems.

646
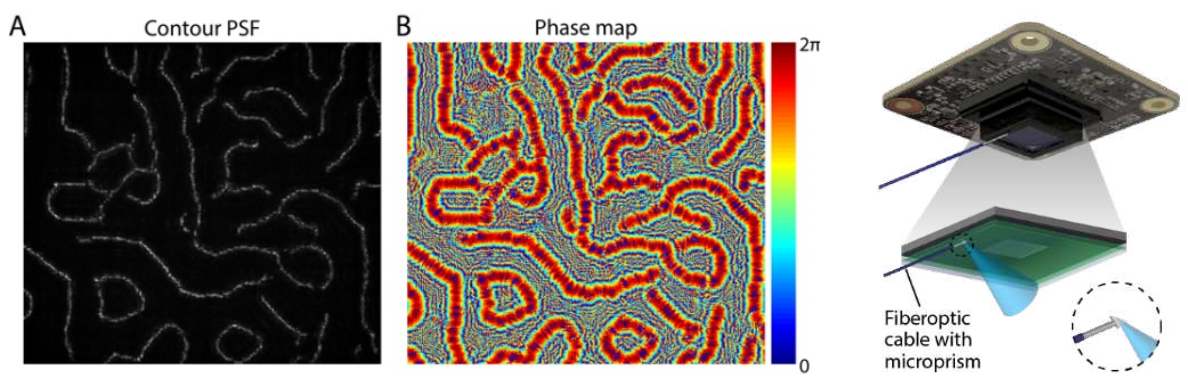

Fig. S2. (A) Contour-based PSF design provides robustness to noise and capturing many directional filters. (B) Phase map for the mask in order to produce the PSF on the image sensor.

Fig. S3. Bio-FlatScope with nearepi illumination with microprism and fiber-optic cable. 
Ground Truth $4 \times$ Objective

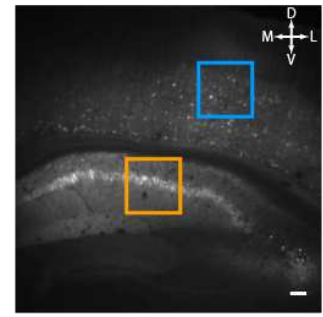

Captured by Bio-FlatScope

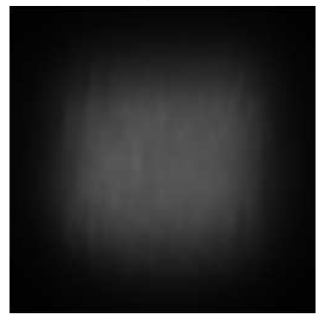

Bio-FlatScope Reconstruction

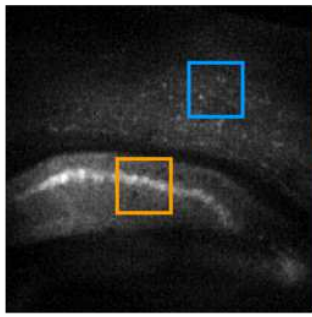

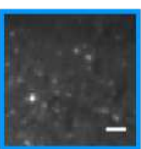
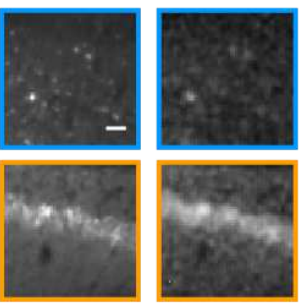

Fig. S4. Mouse brain slice imaging. Ground truth capture, raw capture by Bio-FlatScope, and BioFlatScope reconstruction of a mouse brain slice expressing GCaMP6f. The compass shows dorsal-D, ventral-D, medial-M and later-L directions. Scale bar, $100 \mu \mathrm{m}$. Far right shows zoom-in comparisons of ground truth and Bio-FlatScope reconstructions, respectively. Scale bar, $50 \mu \mathrm{m}$.

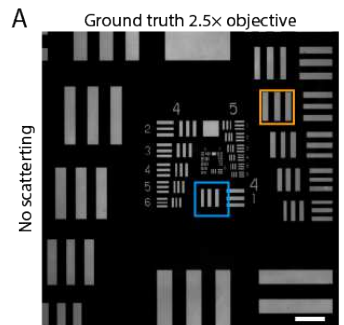

B

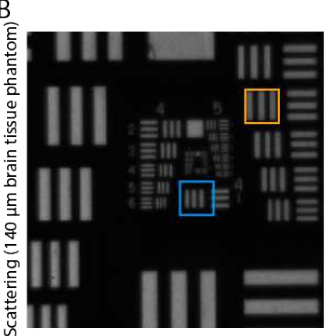

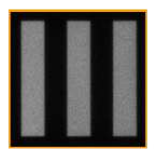
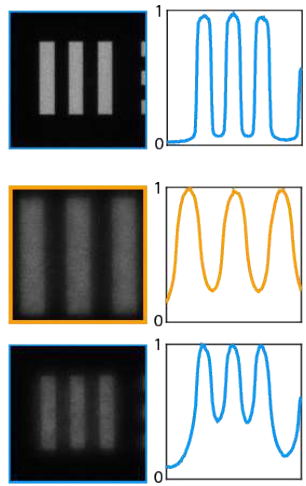

Bio-FlatScope Reconstruction
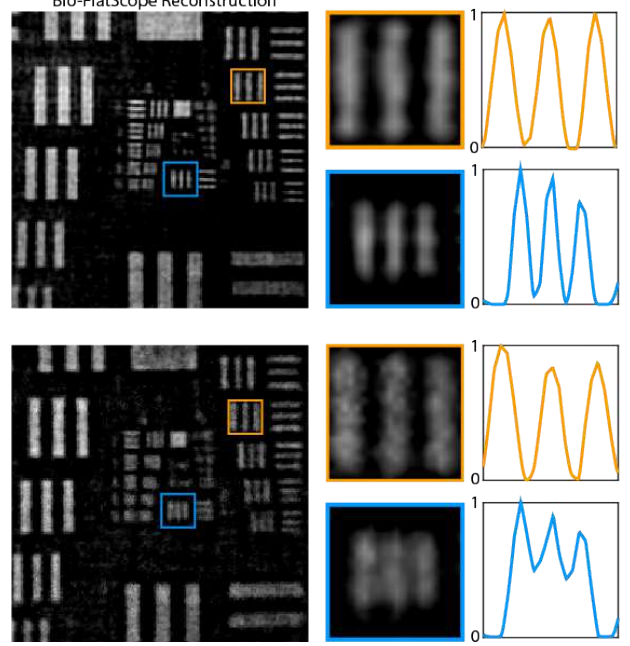
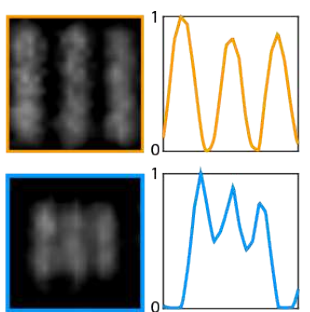

Fig. S5. Imaging through scattering medium. (A) Ground truth (left, captured with a $2.5 \times$ objective and Bio-FlatScope reconstruction (right, captured at $\sim 8.5 \mathrm{~mm}$ from the device) of USAF 1951 resolution target. Scale bar, $250 \mu \mathrm{m}$. Zoom-ins are shown for Group 3 element 3 and group 4 element 1 . Scale bar, $50 \mu \mathrm{m}$. (B) Ground truth (left, captured with a $2.5 \times$ objective) and Bio-FlatScope reconstruction (right, captured at $\sim 8.5 \mathrm{~mm}$ from the device) of USAF 1951 resolution target captured through $140 \mu \mathrm{m}$ of brain tissue phantom. Scale bar, $250 \mu \mathrm{m}$. Zoom-ins are shown for Group 3 element 3 and group 4 element 1 . Scale bar, $50 \mu \mathrm{m}$. 


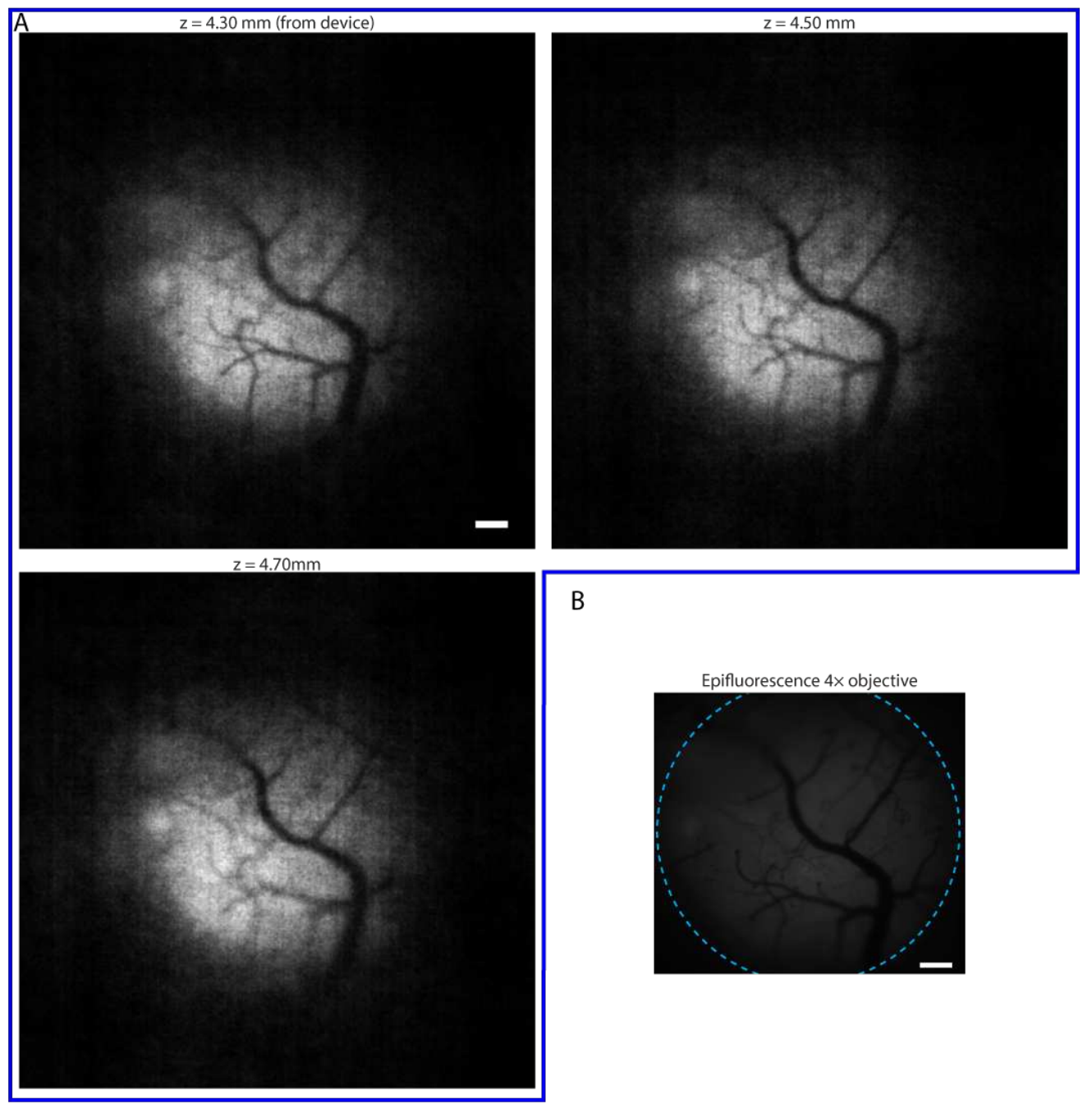

Fig. S6. Full Bio-FlatScope field of view for in vivo mouse brain recordings. (A) Full FOV Bio-FlatScope reconstructions of mouse brain in vivo at $4.3 \mathrm{~mm}, 4.5 \mathrm{~mm}$, and $4.7 \mathrm{~mm}$ from the device. Dark areas (around edges) are regions located outside of the cranial window. Scale bar, $250 \mu \mathrm{m}$ (B) Epifluorescence image of same region of the mouse brain captured with a $4 \times$ microscope objective. Dashed cyan line indicates the outer edge of the cranial window. Scale bar, $250 \mu \mathrm{m}$. 

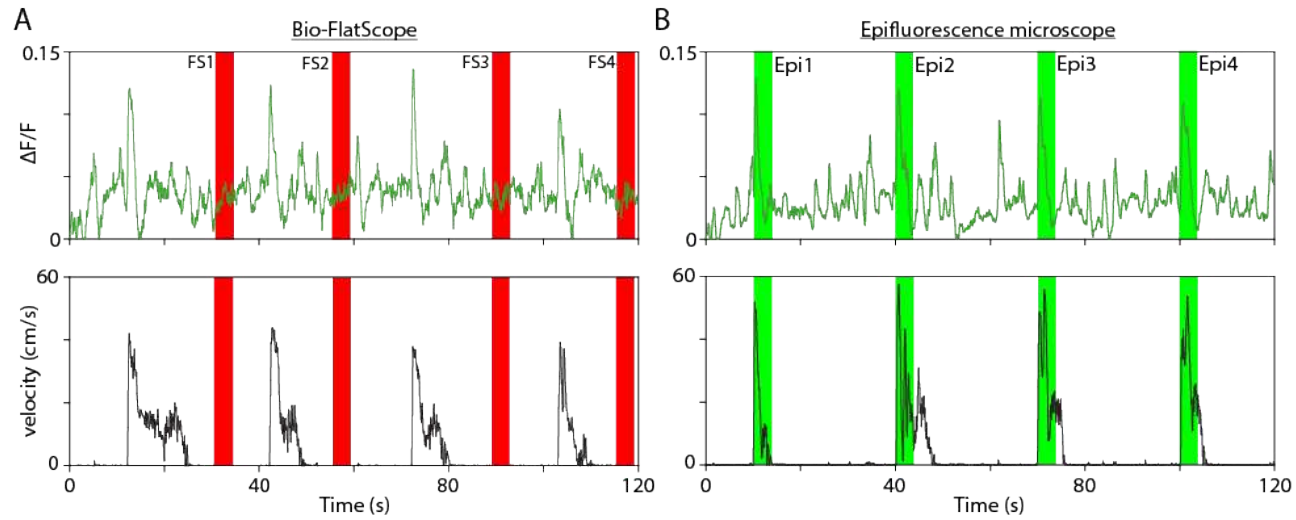

C

$\mathrm{D}$
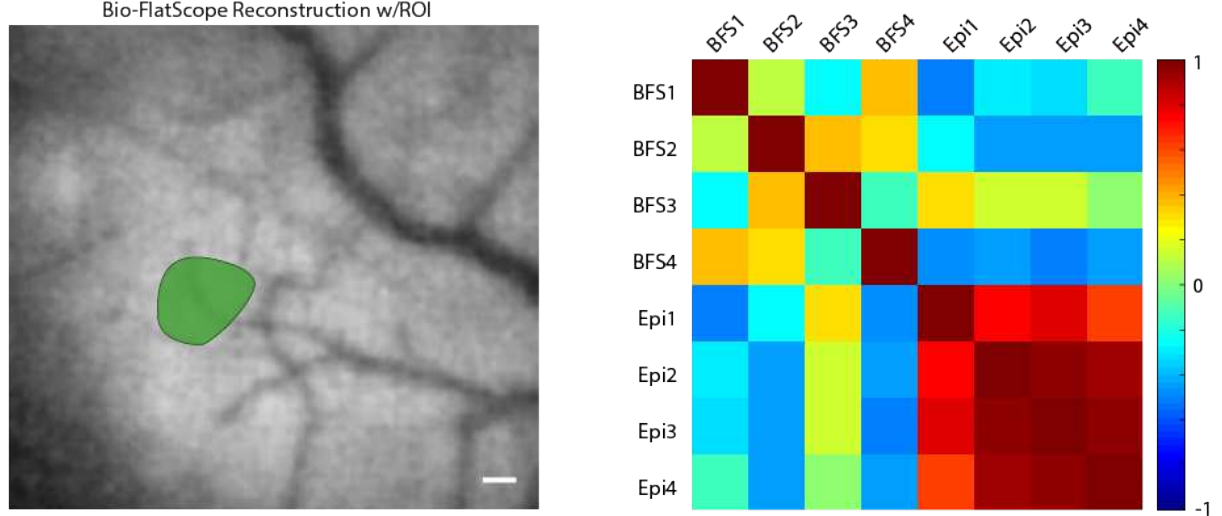

Fig. S7. Comparison of Bio-FlatScope $\mathrm{Ca}^{2+}$ responses during stationary periods to stimulus-evoked responses in epifluorescence recordings. (A) $\Delta \mathrm{F} / \mathrm{F}$ trace and treadmill velocity for Bio-FlatScope during recording session. The 4 -second windows are selected when the mouse is stationary (having a velocity $<$ $1 \mathrm{~cm} / \mathrm{s}$ ), shown in red. (B) $\Delta \mathrm{F} / \mathrm{F}$ trace and treadmill velocity for epifluorescence during recording session. The rising edge of the 4-second windows in green correspond to the application of tactile stimuli. (C) Bio-FlatScope reconstruction with a single ROI of high-activity marked. Scale bar, $100 \mu \mathrm{m}$. (D) Correlation matrix comparing the 4-second windows of little to no movement (red) and stimulus response activity (green). (BFS - Bio-FlatScope, Epi-Epifluorescence microscope). 

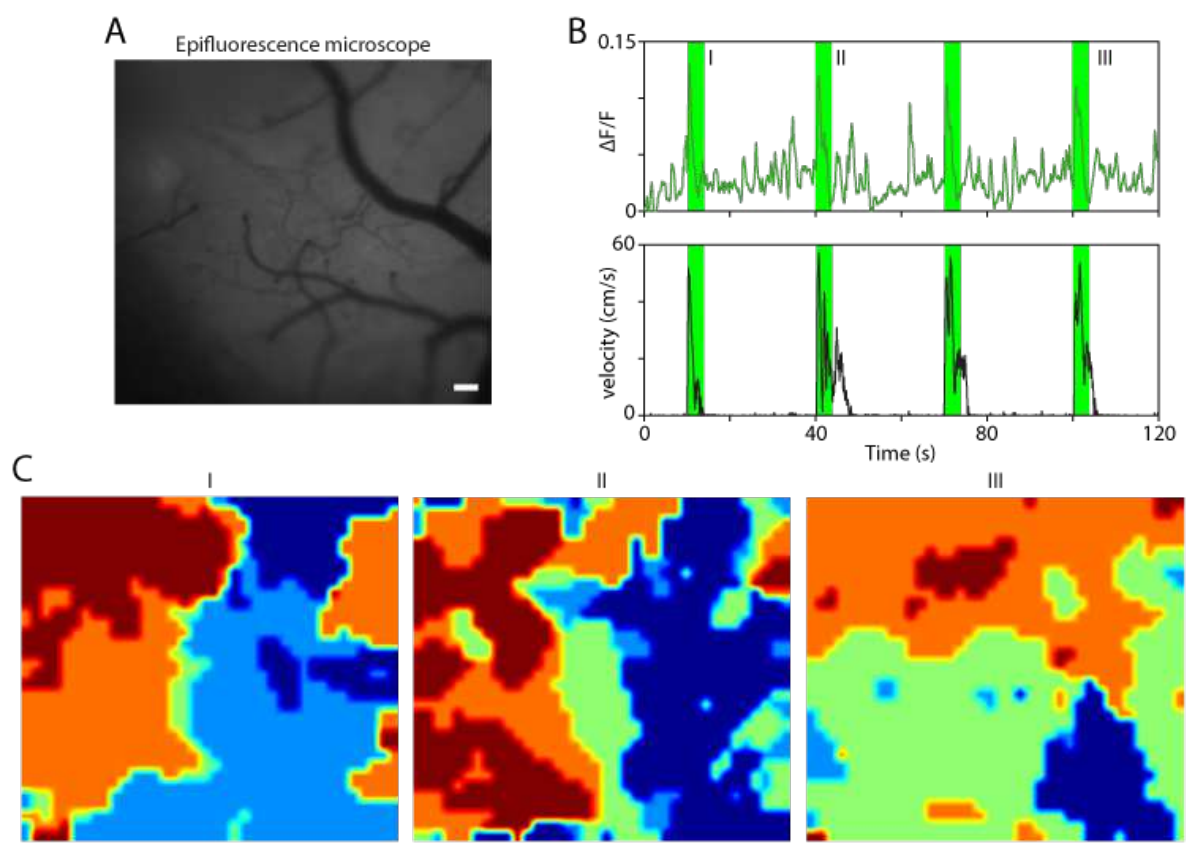

Time (s)

Fig. S8. Spatiotemporal $\mathrm{Ca}^{2+}$ dynamics from epifluorescence recording. (A) Single frame from epifluorescence microscope capture of ROI. Scale bar, $100 \mu \mathrm{m}$. (B) $\Delta \mathrm{F} / \mathrm{F}$ trace and treadmill velocity for epifluorescence during recording session. The rising edge of the 4-second windows in green correspond to the application of tactile stimuli. (C) Heat maps for epifluorescence captures showing spatiotemporal $\mathrm{Ca}^{2+}$ dynamics time-aligned with stimuli (at I, II, and III). Colormap shows the time at which pixels have their peak response for $\Delta \mathrm{F} / \mathrm{F}$ during a $400 \mathrm{~ms}$ period.
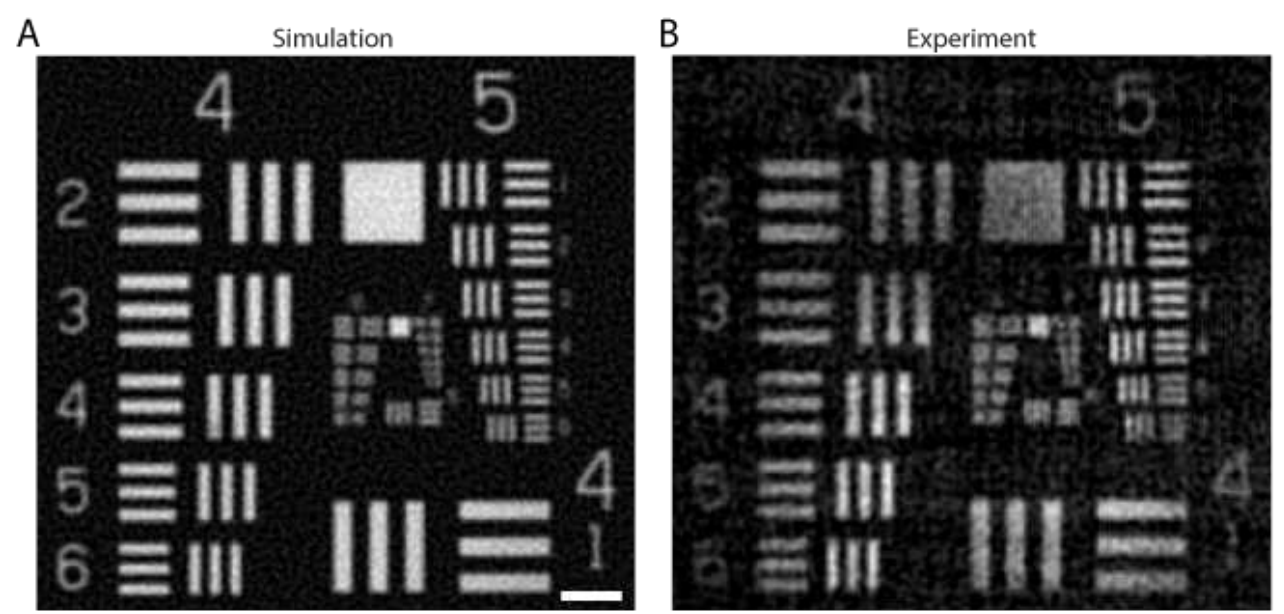

Fig. S9. Simulation vs experimental result of USAF. (A) Simulated Bio-FlatScope reconstruction of USAF target at $4.15 \mathrm{~mm}$ from device. Scale bar, $100 \mu \mathrm{m}$. (B) Experimental Bio-FlatScope reconstruction of USAF target at the same distance from device. The simulation result shows a very close match to the experimental result with group 5 element 6 being resolved. 

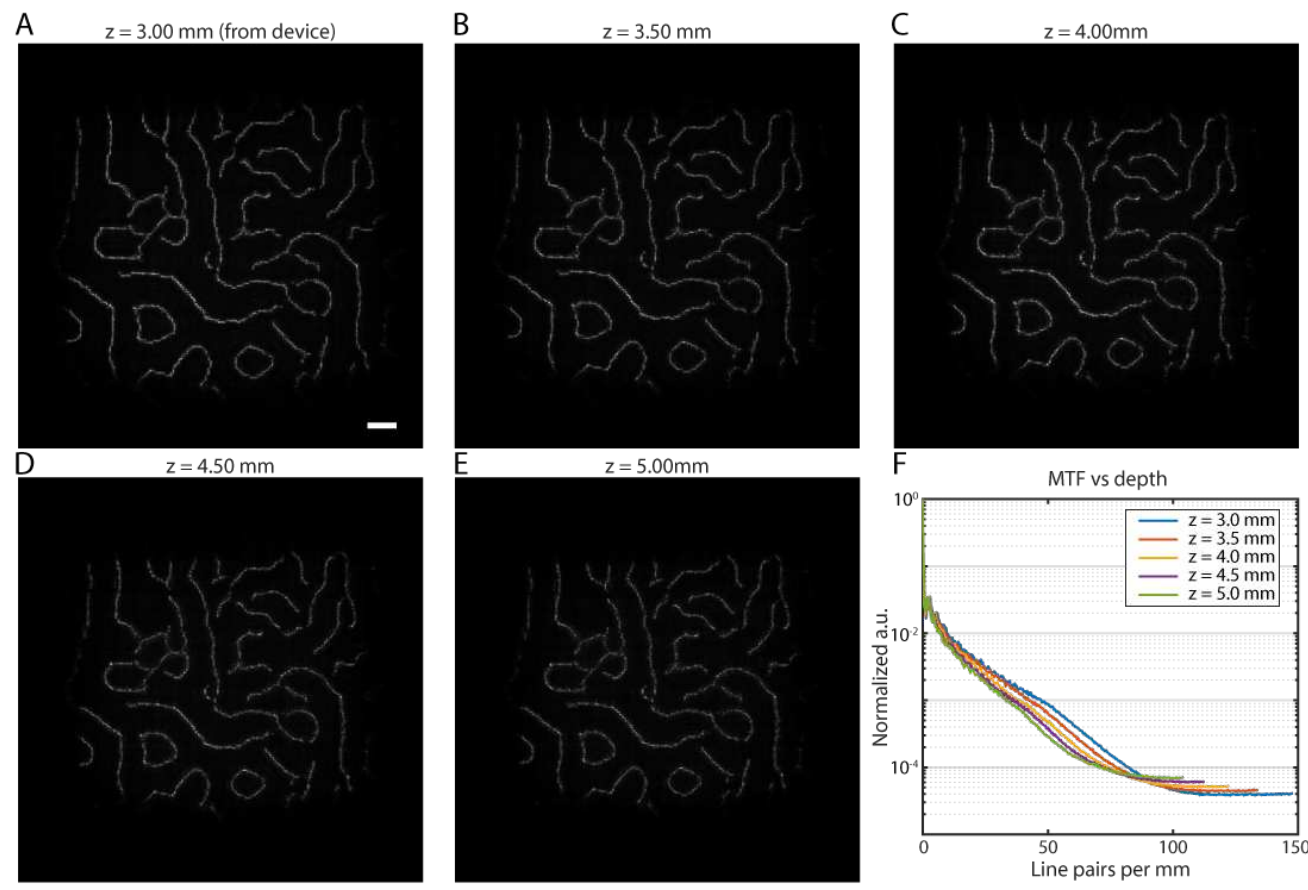

Fig. S10. Captured Bio-FlatScope point spread functions. Bio-FlatScope PSFs captured by imaging a $10 \mu \mathrm{m}$ fluorescent microsphere (see Methods) at different depth planes of (A) $3.00 \mathrm{~mm}$, (B) $3.50 \mathrm{~mm}$, (C) $4.00 \mathrm{~mm}$, (D) $4.50 \mathrm{~mm}$, and (E) $5.00 \mathrm{~mm}$. Scale bar, $100 \mu \mathrm{m}$. (F) Computed MTF from PSFs at different depths from Bio-FlatScope. We observe that the resolution decreases with increasing depth, as seen from the shifting of MTF curves to the left.
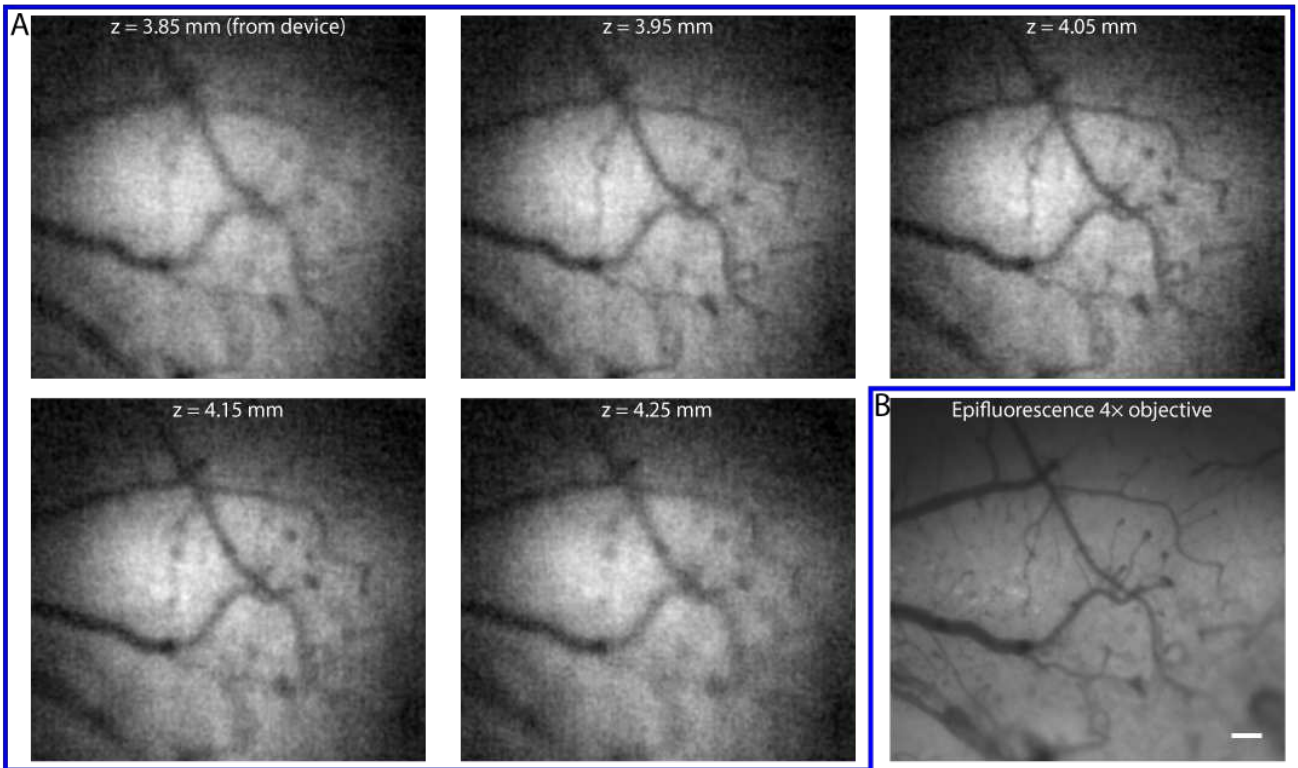

Fig. S11. Digitally refocusing in mouse brain. (A) Bio-FlatScope reconstructions for five different depth planes. At $4.05 \mathrm{~mm}$, the image becomes sharpest for the FOV. (B) Epifluorescence image for the same FOV. Scale bar, $100 \mu \mathrm{m}$. 

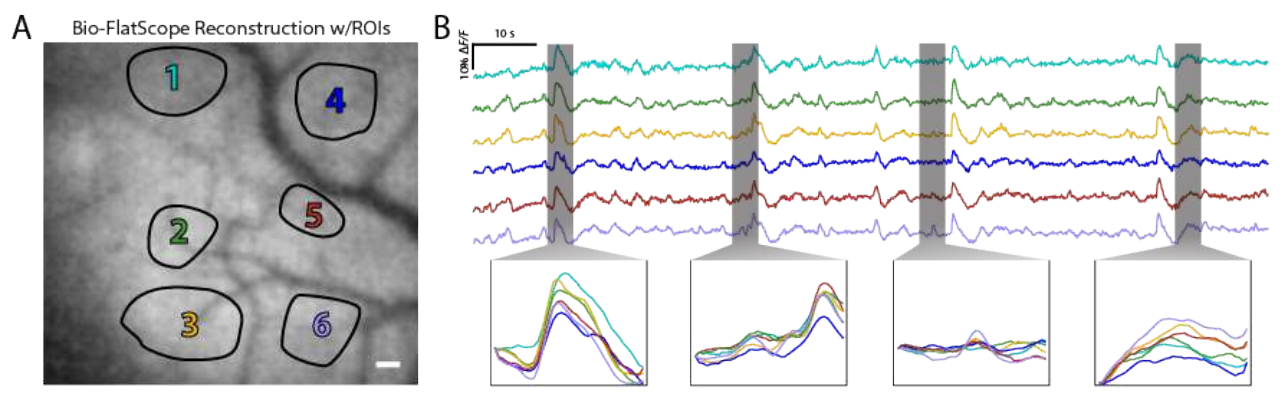

Fig. S12. $\mathrm{Ca}^{2+}$ responses from Bio-FlatScope recording across multiple ROIs. (A) Bio-FlatScope reconstruction showing multiple ROIs. Scale bar $100 \mu \mathrm{m}$. (B) $\Delta \mathrm{F} / \mathrm{F}$ traces for the multiple regions with zoom-ins showing differences in activity across ROIs.

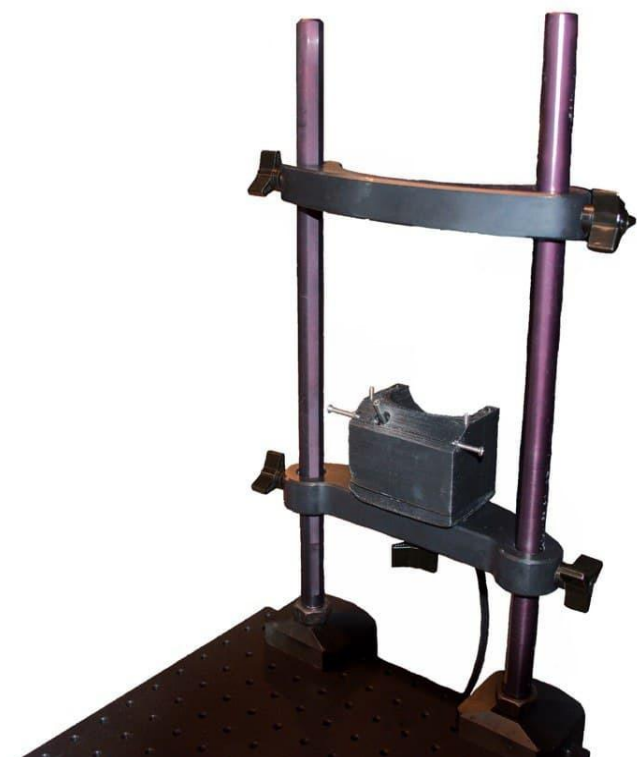

Fig. S13. Oral mucosa imaging setup, including a chin-head rest and a liquid light guide connected to a tabletop LED source for illumination. This photo shows how the liquid light guide is mounted in the chin rest. 
Table S1. Comparison of Bio-FlatScope to miniaturized, head-mounted microscopes.

\begin{tabular}{|l|c|c|}
\hline Microscope ${ }^{\mathrm{a}}$ & Lateral resolution & Field of view \\
\hline Bio-FlatScope & $\sim 8.8 \mu \mathrm{m}$ & $\sim 16.2 \mathrm{~mm}^{2}$ \\
\hline Integrated miniature microscope [5] & $\sim 2.5 \mu \mathrm{m}$ & $0.48 \mathrm{~mm}^{2}$ \\
\hline Fiber bundle microscope [6] & $\sim 4.9 \mu \mathrm{m}$ & $\sim 0.3 \mathrm{~mm}^{2}$ \\
\hline Two-photon miniaturized microscope [7] & $0.64 \mu \mathrm{m}$ & $\sim 0.03 \mathrm{~mm}^{2}$ \\
\hline Miniaturized light-field microscope [8] & $6 \mu \mathrm{m}$ & $\sim 0.42 \mathrm{~mm}^{2}$ \\
\hline
\end{tabular}

${ }^{a}$ Selected microscopes have cellular resolution capability and remain light enough $(<3 \mathrm{~g})$ to be mounted on a mouse and allow free behavior without any support. 


\section{References}

1. J. K. Adams, V. Boominathan, B. W. Avants, D. G. Vercosa, F. Ye, R. G. Baraniuk, J. T. Robinson, and A. Veeraraghavan, "Single-frame 3D fluorescence microscopy with ultraminiature lensless FlatScope," Sci. Adv. 3, e1701548 (2017).

2. N. Antipa, G. Kuo, R. Heckel, B. Mildenhall, E. Bostan, R. Ng, and L. Waller, "DiffuserCam: lensless singleexposure 3D imaging," Optica 5, 1 (2018).

3. Tajima, K., Shimano, T., Nakamura, Y., Sao, M. \& Hoshizawa, T. Lensless light-field imaging with multi-phased fresnel zone aperture. in 2017 IEEE International Conference on Computational Photography, ICCP 2017 Proceedings (Institute of Electrical and Electronics Engineers Inc., 2017). doi:10.1109/ICCPHOT.2017.7951485

4. Stork, D. G. \& Gill, P. R. Optical, Mathematical, and Computational Foundations of Lensless Ultra-Miniature Diffractive Imagers and Sensors. International Journal on Advances in Systems and Measurements 7, 201-208 (2014).

5. K. K. Ghosh, L. D. Burns, E. D. Cocker, A. Nimmerjahn, Y. Ziv, A. E. Gamal, and M. J. Schnitzer, "Miniaturized integration of a fluorescence microscope," Nat Methods 8, 871-878 (2011).

6. M. Kim, J. Hong, J. Kim, and H. Shin, "Fiber bundle-based integrated platform for wide-field fluorescence imaging and patterned optical stimulation for modulation of vasoconstriction in the deep brain of a living animal," Biomed. Opt. Express 8, 2781 (2017).

7. W. Zong, R. Wu, M. Li, Y. Hu, Y. Li, J. Li, H. Rong, H. Wu, Y. Xu, Y. Lu, H. Jia, M. Fan, Z. Zhou, Y. Zhang, A. Wang, L. Chen, and H. Cheng, "Fast high-resolution miniature two-photon microscopy for brain imaging in freely behaving mice," Nat Methods 14, 713-719 (2017).

8. O. Skocek, T. Nöbauer, L. Weilguny, F. Mart'’nez Traub, C. N. Xia, M. I. Molodtsov, A. Grama, M. Yamagata, D. Aharoni, D. D. Cox, P. Golshani, and A. Vaziri, "High-speed volumetric imaging of neuronal activity in freely moving rodents," Nat. Methods 15, 1-4 (2018). 


\section{Figures}
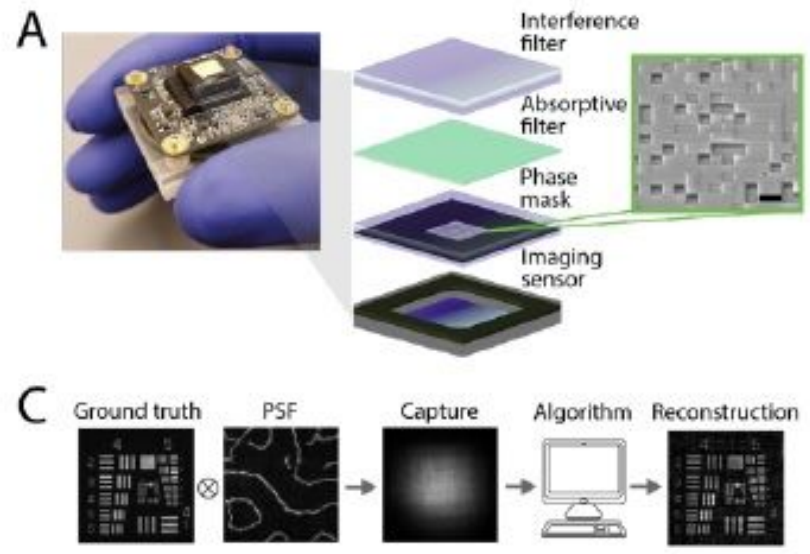

$\mathrm{D}$
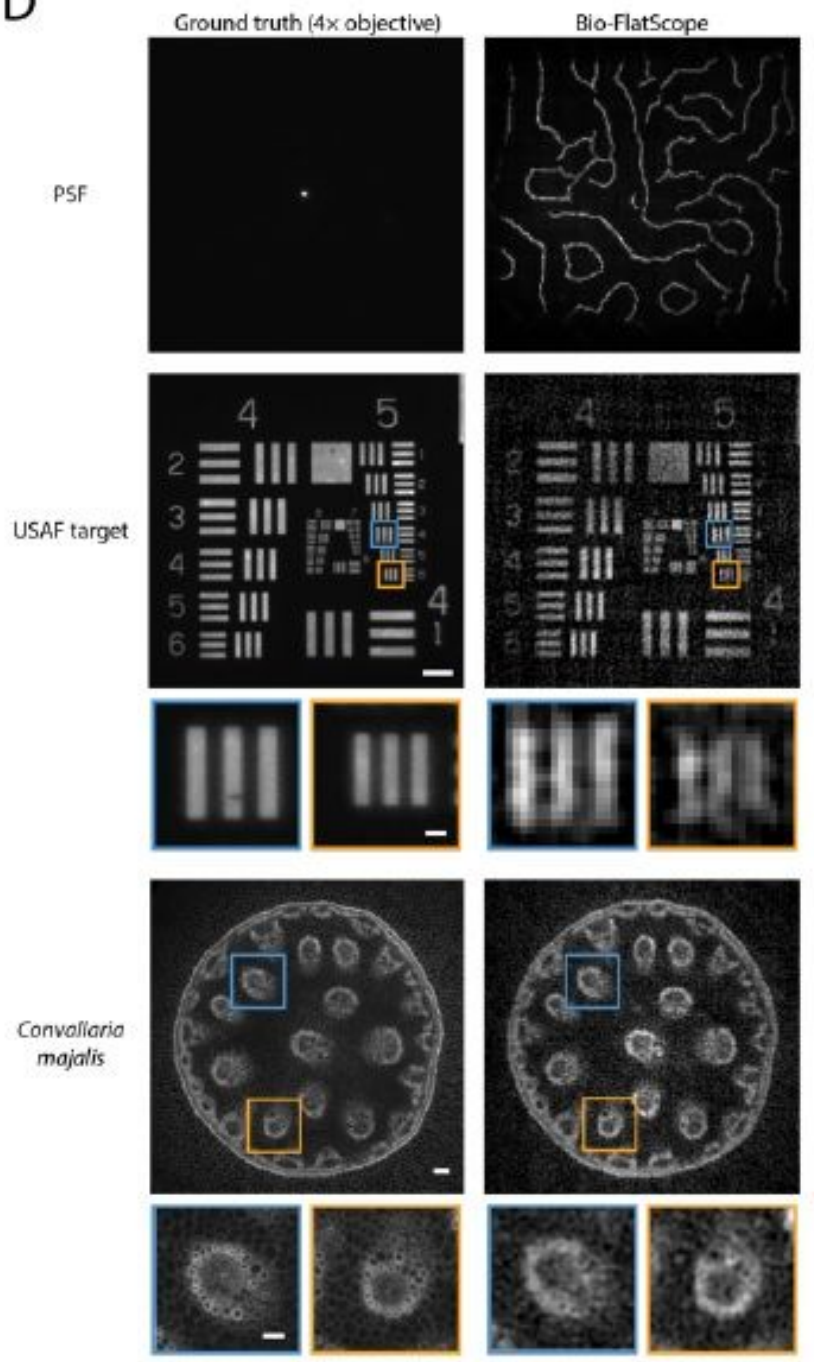
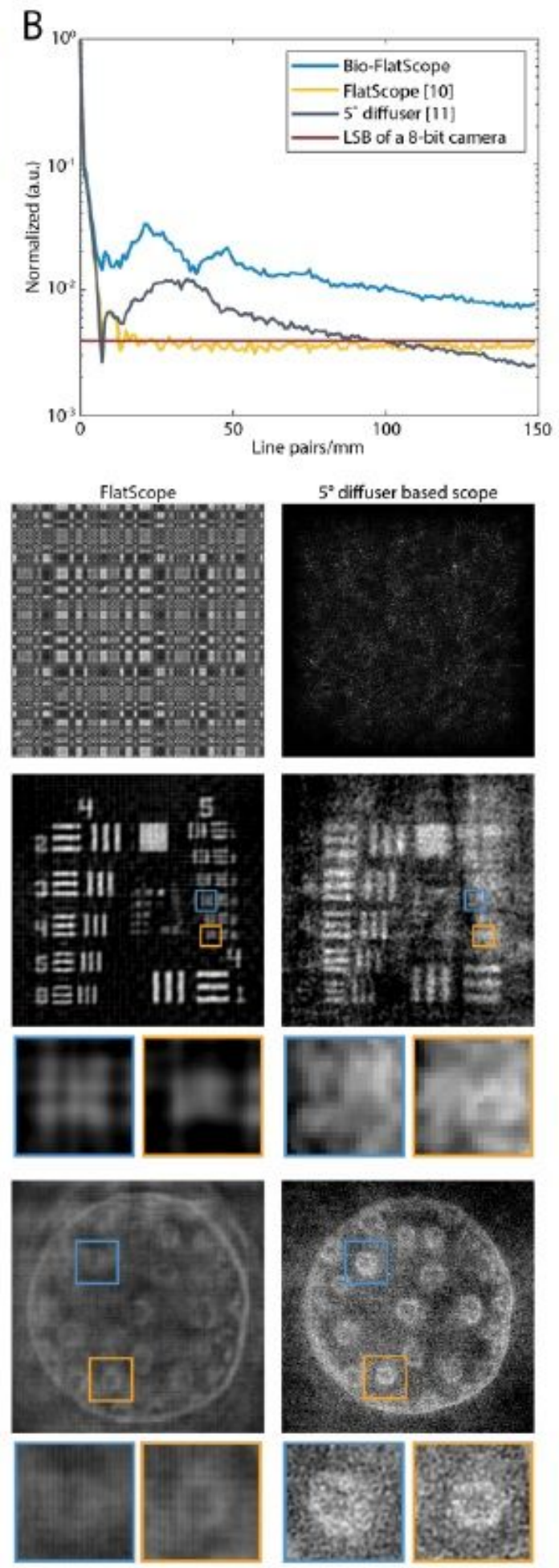

\section{Figure 1}

Bio-FlatScope for in vivo fluorescence imaging. (A) Photo of a Bio-FlatScope prototype including the offthe-shelf board level camera. Zoom-in shows components that filter excitation light and apply the phase mask transfer function to the incident light. Also shown is a scanned electron micrograph of a 
portion of the fabricated phase mask. Scale bar $4 \mu \mathrm{m}$. (B) Modulation transfer function (MTF) comparison among PSF designs used by lensless imaging systems show that the contour PSF used for the Bio-FlatScope contains more high-frequency components, which contributes to improved performance. LSB: least significant bit. (C) Flow chart showing the imaging procedure of Bio-FlatScope. (D) High spatial resolution images of a USAF test target and fixed biological samples. Row 1: PSF captures of different lensless imaging systems. Row 2: Ground truth captured by an epifluorescence microscope and reconstructed by 3 different lensless imaging systems. While FlatScope and BioFlatScope both show good performance for the high-contrast USAF target, only the Bio-FlatScope performs well on the low-contrast, dense Convallaria sample. USAF 1951 Resolution test target scale bar, $100 \mu \mathrm{m}$. Insets below show zoom-ins of group 5, elements of $4 \& 6$. Scale bar, $10 \mu \mathrm{m}$. Convallaria majalis scale bar, $100 \mu \mathrm{m}$. Insets below show zoom-in comparisons. Scale bar, $50 \mu \mathrm{m}$.

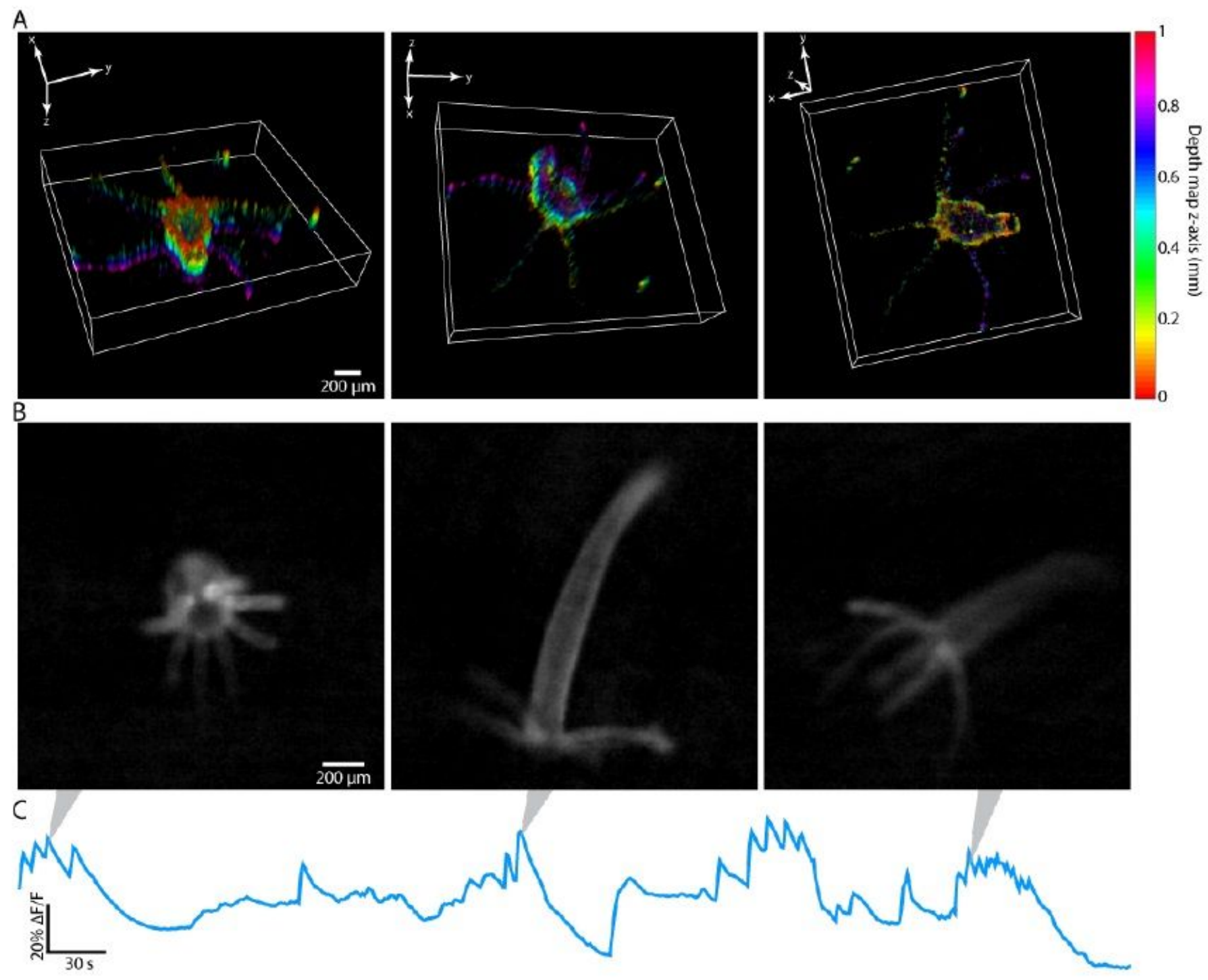

Figure 2

Ca2+ imaging (GCaMP7b) and three-dimensional imaging (GFP) of Hydra vulgaris in vivo. (A) Select angles of three-dimensional Bio-FlatScope reconstructions of Hydra vulgaris expressing GFP in the 
interstitial cell lineage. Colors represent relative depth location along the z-axis. Scale bar, $200 \mu \mathrm{m}$. (B) Selected Bio-FlatScope reconstructed frames of video of Hydra vulgaris expressing GCaMP7b in muscle cells. Scale bar, $200 \mu \mathrm{m}$. (C) $\Delta \mathrm{F} / \mathrm{F}$ traces showing the Ca2+ responses over a 5-minute recording.
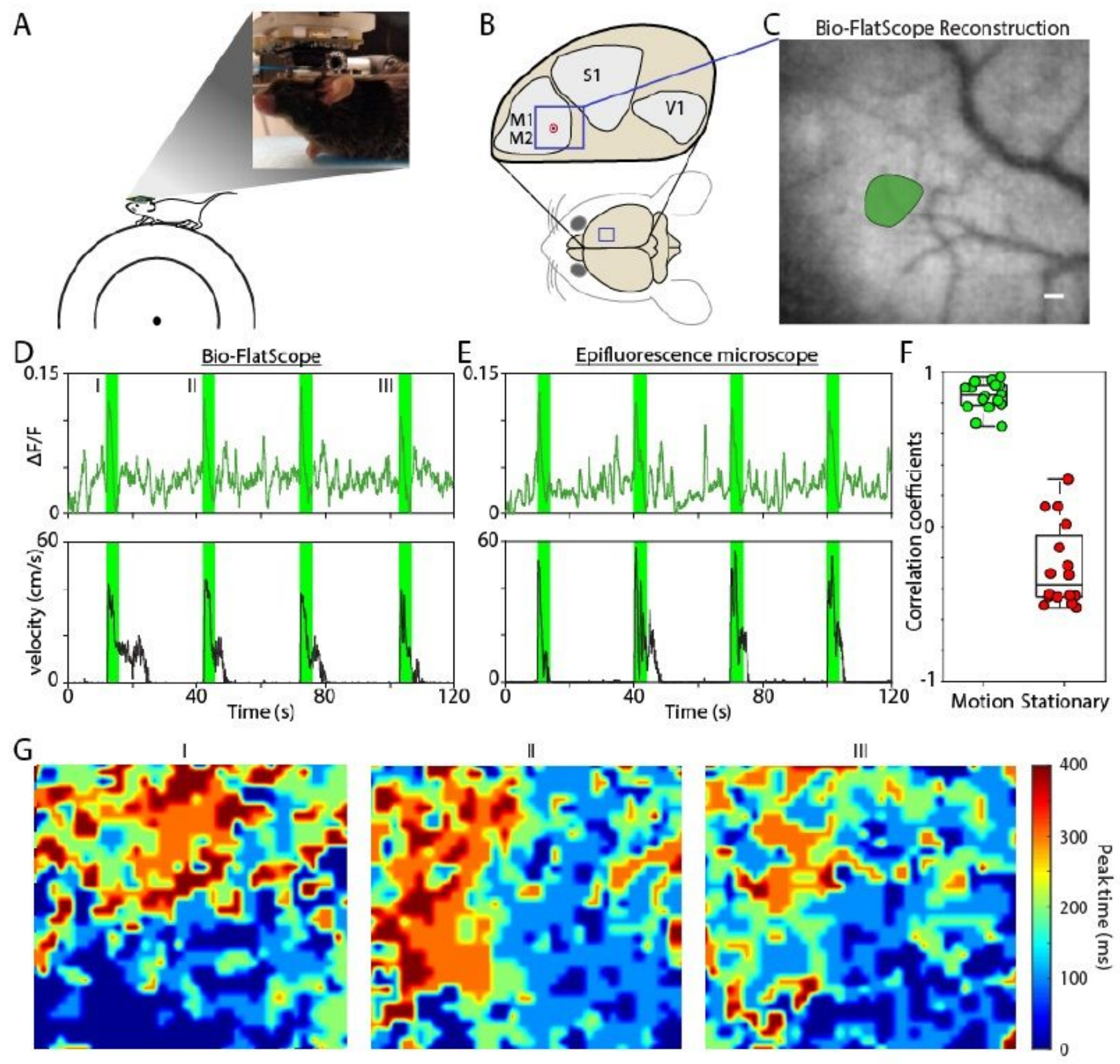

Figure 3

Comparison of Bio-FlatScope to epifluorescence during stimulus-evoked $\mathrm{Ca} 2+$ responses in motor cortex. (A) Experimental setup for Bio-FlatScope for imaging a head-fixed mouse on a freely moving treadmill. Zoom-in shows a photo of a mouse on the treadmill with Bio-FlatScope in place (B) Target region in the mouse brain. The blue square region indicates the approximate location of the cropped FOV (in the motor cortex). The circle with the black dot indicates the approximate region of injection for GCaMP6f. (C) BioFlatScope reconstruction with a single ROI of high-activity marked. Scale bar, $100 \mu \mathrm{m}$. (D) $\Delta \mathrm{F} / \mathrm{F}$ trace and 
treadmill velocity for Bio-FlatScope during recording session. The rising edge of the 4-second windows in green correspond to the application of tactile stimuli. (E) $\Delta F / F$ trace and treadmill velocity for epifluorescence during recording session. (F) Box plot showing correlation coefficients comparing stimulus-evoked Ca2+ responses from Bio-FlatScope to epifluorescence (shown in green), and comparison of periods of little or no motion with Bio-FlatScope to stimulus-evoked Ca2+ responses with epifluorescence (shown in red) for the ROI. (G) Heat maps for Bio-FlatScope reconstructions showing spatiotemporal Ca2+ dynamics time-aligned with stimuli (at I, II, and III). Colormap shows the time at which pixels have their peak response for $\Delta F / F$ during a $400 \mathrm{~ms}$ period. These data show that when initiating movement, neural activity consistently begins in the lower right and propagates to the upper left.
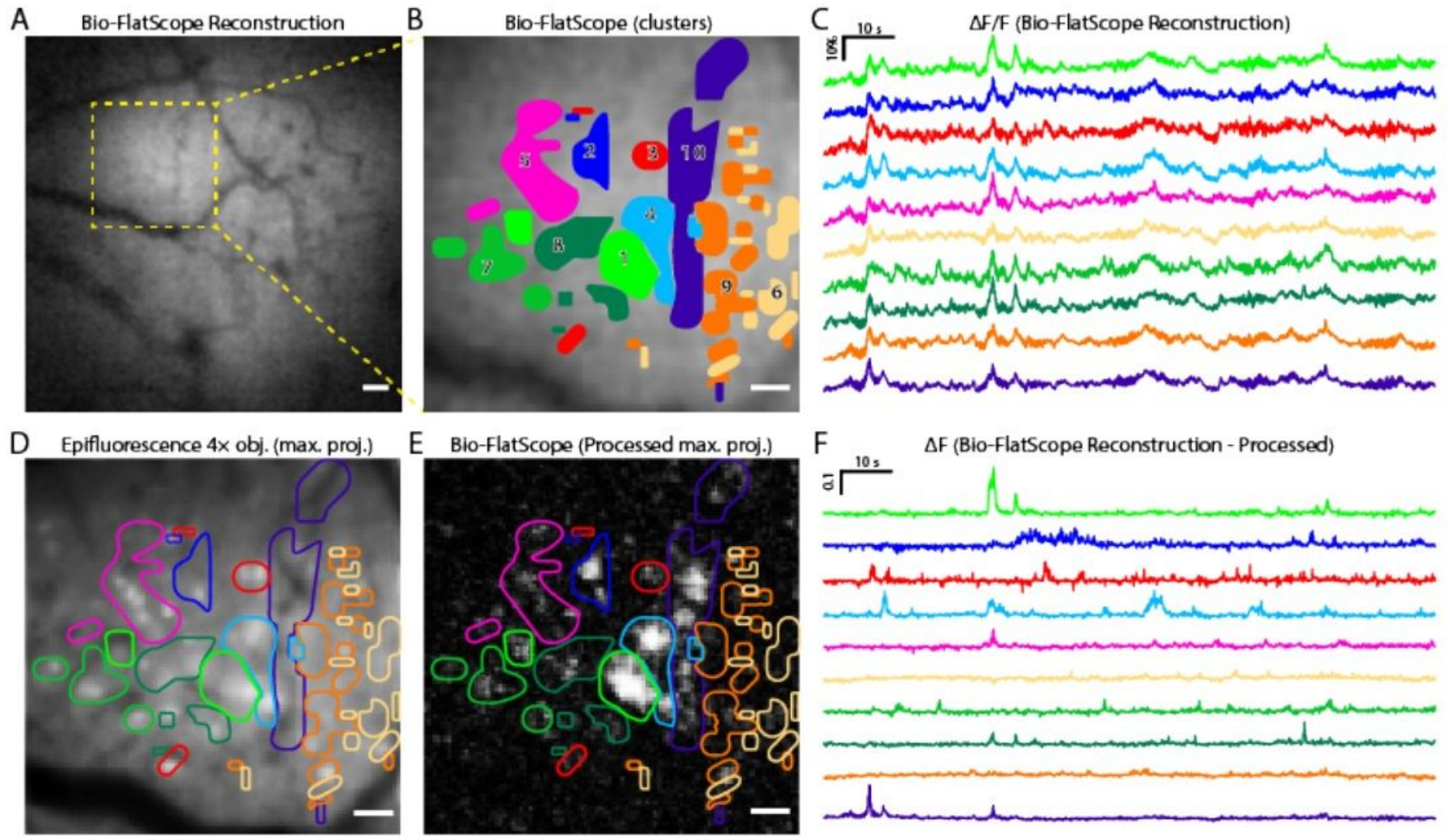

\section{Figure 4}

Extracting near cellular-sized Ca2+ signals from Bio-FlatScope reconstructions. (A) Cropped region of BioFlatScope reconstruction of a single frame with a high-activity region shown by a dashed box. Scale bar, $100 \mu \mathrm{m}$. (B), Zoom-in on the region of high-activity with overlay of clusters determined through postprocessing using RPCA and k-means. Scale bar, $50 \mu \mathrm{m}$. (C) $\Delta \mathrm{F} / \mathrm{F}$ traces from Bio-FlatScope reconstruction video over during twominute recording corresponding to the clusters. (D) Maximum projection from epifluorescence recording of same high activity region with overlay of clusters determined from Bio-FlatScope data. Scale bar 50 um. (E) Maximum intensity projection of Bio-FlatScope reconstruction data processed using RPCA with overlay of clusters. Scale bar $50 \mu m$. (F) $\Delta F$ traces from Bio-FlatScope reconstructions after post-processing using RPCA. 
A
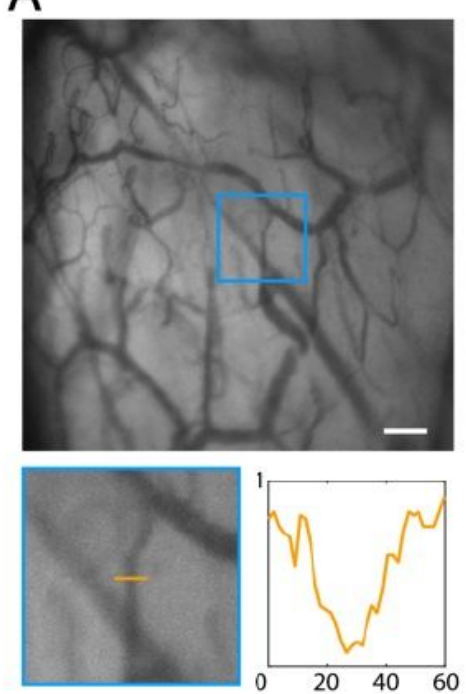

C

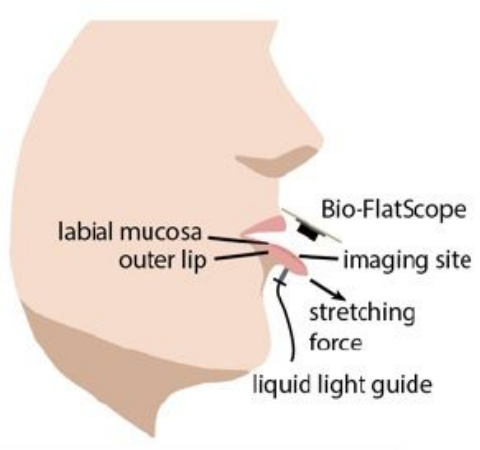

B
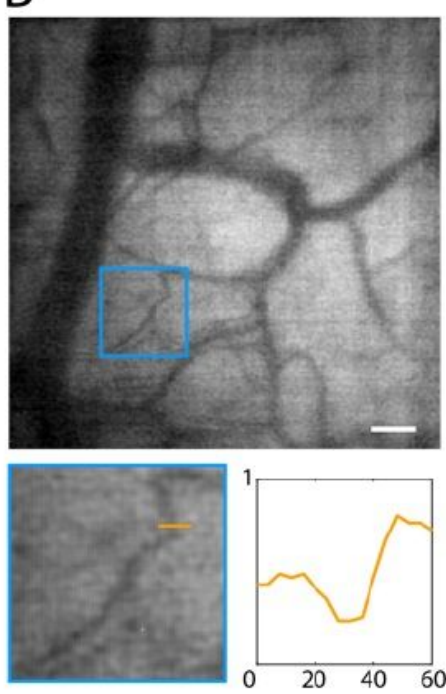

D

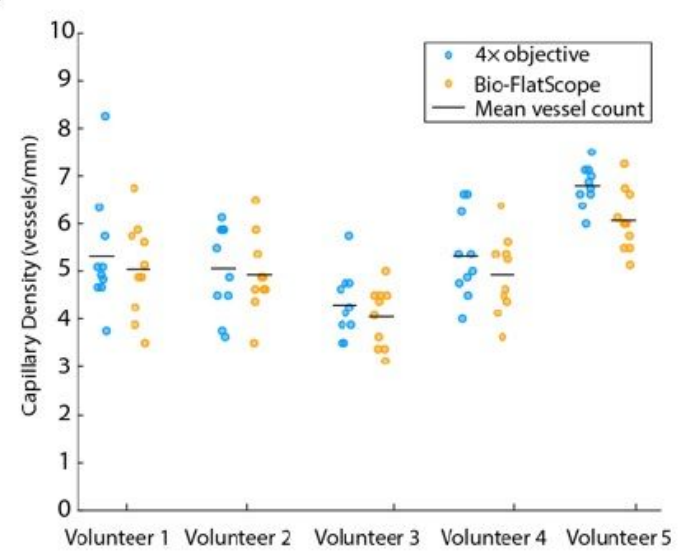

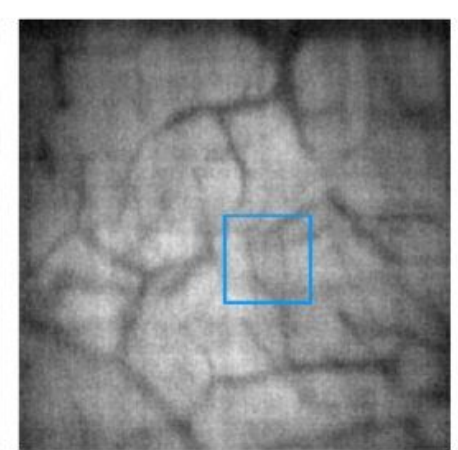
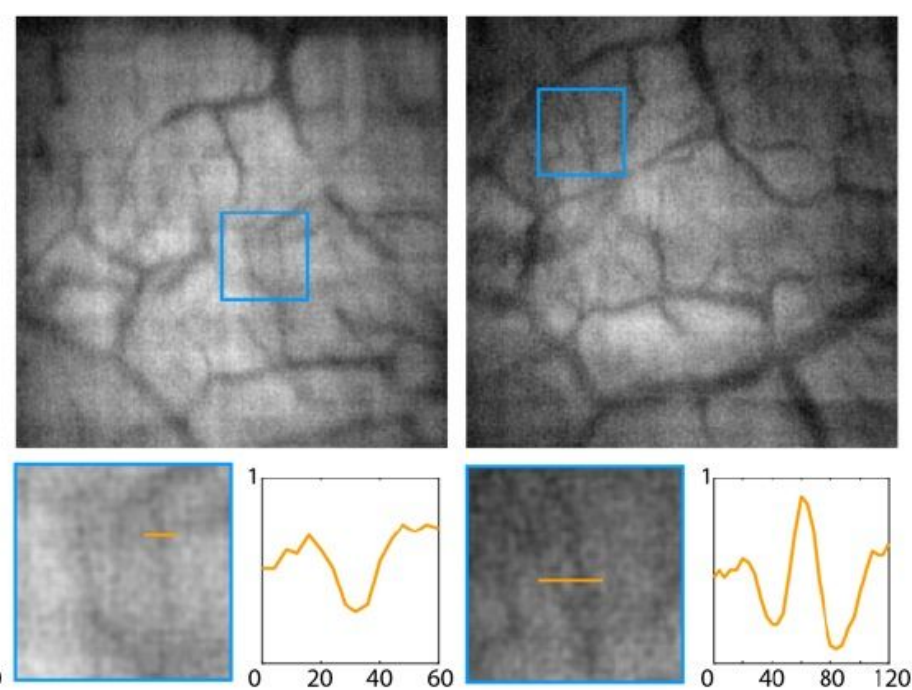

$\mathrm{E}$

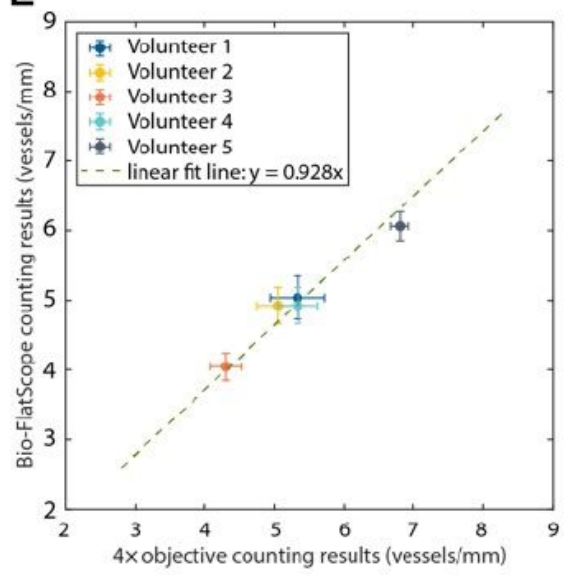

\section{Figure 5}

Human oral mucosa imaging. (A) Images acquired using a 4x objective. Scale bar, $200 \mu \mathrm{m}$.

(B) Reconstructed images from a Bio-FlatScope prototype. Each blue square indicates a sub-region which contains $\sim 15 \mu \mathrm{m}$ diameter vessels. Scale bar, $200 \mu \mathrm{m}$. Left insets, zoom-ins of sub-regions. Right insets, pixel intensity along the cross section (shown as orange bars) of the vessels. Length of orange bars in insets, $60 \mu \mathrm{m}(120 \mu \mathrm{m}$ in the 4th inset). X-axis: distance (in $\mu \mathrm{m})$ from the left origin of the orange bar. Yaxis: Pixel intensity in normalized arbitrary units, shared by all 4 plots. (C) Experimental setup. The transmissive illumination is delivered by a liquid light guide connected to a green LED light source. (D) Vessel counting results of each volunteer. Each outlined circle corresponds to a different region on the lip. Less translucency indicates overlapping dots. There are 10 circles in each column. (E) Relationship between 4× objective and Bio-FlatScope counting results. Each circle is the average of 10 different lip regions as shown in panel $D$. Note that we imaged the same approximate of the labial mucosa with both the $4 \mathrm{X}$ and Bio-FlatScope; however, were unable to capture images of the exact same area of tissue when switching between imaging modalities. The error bars show the standard error of the mean.

\section{Supplementary Files}


This is a list of supplementary files associated with this preprint. Click to download.

- SupplementaryinformationBioFlatScopemanuscriptsubmitted01062021.pdf

- SupplementaryVideo1HydravulgarisGCamp7b.mov

- SupplementaryVideo2MouseCortexGCaMP6f.mov 